\title{
Convergence Analysis of a Domain Decomposition Paradigm
}

\author{
Dedicated to Professor Wolfgang Hackbusch \\ on the occasion of his 60th birthday
}

\begin{abstract}
We describe a domain decomposition algorithm for use in several variants of the parallel adaptive meshing paradigm of Bank and Holst. This algorithm has low communication, makes extensive use of existing sequential solvers, and exploits in several important ways data generated as part of the adaptive meshing paradigm. We show that for an idealized version of the algorithm, the rate of convergence is independent of both the global problem size $N$ and the number of subdomains $p$ used in the domain decomposition partition. Numerical examples illustrate the effectiveness of the procedure.
\end{abstract}

Keywords Domain decomposition, Bank-Holst algorithm, parallel adaptive grid generation

Mathematics Subject Classification (2000) 65N55, $65 \mathrm{~N} 50$

\section{Introduction}

In $[7,8]$, Bank and Holst introduced a general approach to parallel adaptive meshing for systems of elliptic partial differential equations. This approach was motivated by the desire to keep communications costs low, and to allow sequential adaptive software (such as the software package PLTMG [4] used in this work) to be employed without extensive recoding.

The work of R. Bank was supported by the National Science Foundation under contract DMS-0511766. The UCSD Scicomp Beowulf cluster was built using funds provided by the National Science Foundation through SCREMS Grant 0112413, with matching funds from the University of California at San Diego.

The work of P. Vassilevski was performed under the auspices of the U. S. Department of Energy by the University of California Lawrence Livermore National Laboratory under contract W-7405-Eng-48.

Bank: Department of Mathematics, University of California, San Diego, La Jolla, California 92093-0112. Email: rbank@ucsd.edu.

Vassilevski: Center for Applied Scientific Computing, Lawrence Livermore National Laboratory, Livermore CA 94550. Email: panayot@1lnl.gov.
The original paradigm has three main components:

Step I: Load Balancing. We solve a small problem on a coarse mesh, and use a posteriori error estimates to partition the mesh. Each subregion has approximately the same error, although subregions may vary considerably in terms of numbers of elements or gridpoints.

Step II: Adaptive Meshing. Each processor is provided the complete coarse mesh and instructed to sequentially solve the entire problem, with the stipulation that its adaptive refinement should be limited largely to its own partition. The target number of elements and grid points for each problem is the same. At the end of this step, the mesh is regularized such that the global mesh described in Step III is conforming.

Step III: Global Solve. The final global mesh consists of the union of the refined partitions provided by each processor. A final solution is computed using domain decomposition.

With this paradigm, the load balancing problem is reduced to the numerical solution of a small elliptic problem on a single processor, using a sequential adaptive solver such as PLTMG without requiring any modifications to the sequential solver. The bulk of the calculation in the adaptive meshing step also takes place independently on each processor and can also be performed with a sequential solver with no modifications necessary for communication. The only parts of the calculation requiring communication are:

- The initial fan-out of the mesh distribution to the processors at the beginning of Step II, once the decomposition is determined by the error estimator in load balancing.

- Mesh regularization at the end of Step II requires communication to produce a global conforming mesh.

- The domain decomposition solver in Step III requires communicating certain information about the interface system.

In [6], Bank considered a variant of the above approach in which the load balancing occurs on a much finer mesh. The motivation was to address some possible problems arising from the use of a coarse grid in computing the load balance. This variant also has three main components. 
Step I: Load Balancing. On a single processor we adaptively create a fine mesh of size $N_{p}$, and use a posteriori error estimates to partition the mesh such that each subregion has approximately equal error, similar to Step I of the original paradigm.

Step II: Adaptive Meshing. Each processor is provided the complete adaptive mesh and instructed to sequentially solve the entire problem. However, in this case each processor should adaptively coarsen regions corresponding to other processors, and adaptively refine its own subregion. The size of the problem on each processor remains $N_{p}$, but this adaptive rezoning strategy concentrates the degrees of freedom in the processor's subregion. At the end of this step, the mesh is regularized such that the global mesh is conforming.

Step III: Global Solve. This step is the same as in the original paradigm.

Using the variant, the initial mesh can be of any size. Indeed, our choice of $N_{p}$ is mainly for convenience and to simplify notation; any combination of coarsening and refinement could be allowed in Step II. Allowing the mesh in Step I to be finer increases the cost of both the solution and the load balance in Step I, but it allows flexibility in overcoming potential deficiencies of a very coarse mesh in the original paradigm. See $[7,8,11]$ for numerical examples of the original paradigm and $[6,5]$ for examples comparing the original and variant paradigms.

Although both the original paradigm and the variant use the same domain decomposition solver in Step III, the variant algorithm produced some unforeseen consequences. In particular, in the PLTMG package, in Step II of the paradigm, edges lying on the interface system can be refined as necessary. Vertices added during refinement steps can be deleted during coarsening steps, but the original vertices defining the interface system must remain in the mesh during Steps II and III of either paradigm. This restriction insures that the subdomains remain geometrically conforming across all processors, and also plays an important role in the mesh regularization algorithm applied at the end of Step II.

This point is of little consequence for the original paradigm because it is based mainly on refinement. However, it is quite significant for the variant. Indeed, for the variant, coarsening is limited to the interiors of subdomains corresponding to other processors, while the parts of the interface system lying in the coarse parts of the domain remain largely unchanged. Thus in the domain decomposition solver the local problem has an unusual structure, in that it is highly refined on its own subdomain and its part of the interface system, it is very coarse in the interior of other subregions, and it has the original level of refinement on the coarse parts of the interface system.

The situation is illustrated in Figure 1. Here a square domain $\Omega$ is partitioned into $p=4$ subdomains. We illustrate the mesh as it exists on a typical processor at the end of Step II. In the original paradigm, shown on the left, the mesh is (adaptively) refined in one subregion (shaded gray and labeled $h$ ) and remains coarse in the other three regions (la-
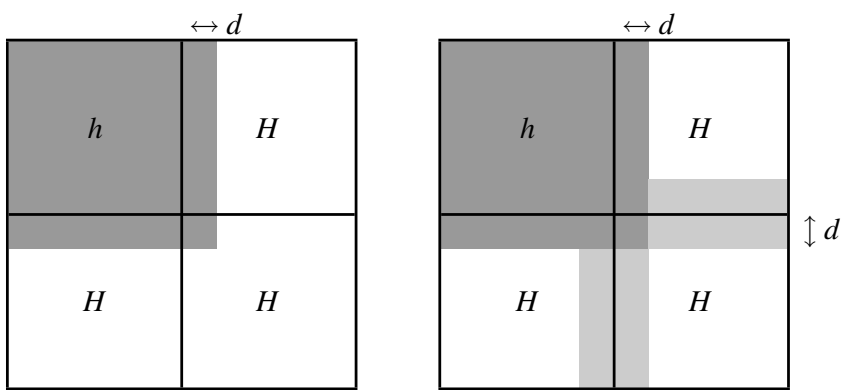

Fig. 1 In this figure $\Omega$ is a square domain, partitioned into $p=4$ subdomains. We illustrate the situation on one processor at the end of Step II for the original paradigm (left) and the variant (right).

beled $H$ ). Due to constraints of shape regularity, the refined region bleeds into the coarse region in order to smoothly grade the elements from small to large giving a band of refined elements of width $d$. For the variant algorithm, shown of the right, the mesh remains fine along the interface because vertices on the interface cannot be unrefined. Thus there are bands of refined elements along the interfaces in the coarse region as well (colored light gray in Figure 1). Due to shape regularity constraints, the fine mesh at the interface is graded into a mesh of larger elements in the interiors of the coarse subregions. Our analysis here led to a heuristic for refining the coarse interface in the case of the original paradigm as part of the mesh regularization. This is done to improve the robustness of the domain decomposition solver, and is described in more detail in Section 6.

The purpose of this work is to analyze the domain decomposition solver in the environment provided by these parallel adaptive refinement strategies. This solver reflects the philosophy of the adaptive meshing paradigm in that communication costs are low. In our algorithm, each processor solves a sequence of global linear systems corresponding to the regularized conforming grids that are created at the conclusion of Step II. The right hand sides are chosen such that these solutions converge to the global conforming finite element solution on the fine portion of each processor's mesh. Since each processor solves global problems, no special global coarse mesh solves are required. For an idealized version of the algorithm we are able to show that the rate of convergence is independent of both the global problem size $N$ and $p$. See [29,17,27,33] for general background on domain decomposition methods. Some discussion of the method developed here and its predecessors can be found in $[10,9,11,21,5]$, while related ideas in the multigrid context can be found in Mitchell [23-25].

Our analysis here is interesting for several reasons. First, the overall iteration does not have a symmetric error propagator, even in the case where the underlying continuous problem and its conforming finite element discretization are self-adjoint and positive definite. Thus we do not take an approach based on estimating generalized condition numbers, but rather make direct norm estimates for the error reduc- 
tion. For a special case, we can frame the analysis in terms of a norm estimate for a symmetric indefinite matrix.

Second, while the approximate solution of the global problem belongs to a usual, globally conforming, finite element space, (in our case, continuous piecewise linear finite elements on a shape regular triangulation) the domain decomposition iteration itself is based on a saddle point formulation for nonconforming finite element spaces. The Lagrange multipliers, which are used to impose continuity at vertices along the interface, have the flavor of Dirac delta functions when viewed in the finite element context. An additional complication in the analysis arises from the fact that these Lagrange multipliers are not actually computed or updated as part of the iteration. Our saddle point formulation of the global system of equations can be viewed as a special (and very simple) example of a mortar element method [14, $13,16,32,31,20]$.

Another part of the analysis draws heavily upon interior estimates for finite element solutions. Such estimates have a long history; see for example the survey by Wahlbin [30]; see also [26,34,2]. Many of the techniques and much of the analysis are now quite standard. Our analysis also has some similarities to that of meshless methods $[22,1]$.

The remainder of the paper is organized as follows. In Section 2, we present our parallel domain decomposition solver using traditional finite element spaces and notation. In Section 3, we compute the error propagator using linear algebra and matrix/operator notation. In Section 4 we provide norm estimates for the rate of convergence in a special case. These estimates are seen to be independent of $N$ and $p$ for the problems considered here. In Section 5, we discuss the practical implementation of the algorithm, in particular the derivation of the symmetric, positive definite systems that are solved on each processor and the parallel communication requirements. Finally, in Section 6 we provide some numerical results.

\section{Preliminaries}

Let $\Omega=\cup_{i=1}^{p} \Omega_{i} \subset \mathscr{R}^{2}$ denote the domain, decomposed into $p$ geometrically conforming subdomains. Let $\Gamma$ denote the interface system. The degree of a vertex $x$ lying on $\Gamma$ is the number of subregions for which $x \in \bar{\Omega}_{i}$. A cross point is a vertex $x \in \Gamma$ with degree $(x) \geq 3$. We assume that the maximal degree at cross points is bounded by the constant $\delta_{0}$. The connectivity of $\Omega_{i}$ is the number of other regions $\Omega_{j}$ for which $\bar{\Omega}_{i} \cap \bar{\Omega}_{j} \neq \emptyset$. We assume the connectivity of $\Omega_{i}$ is bounded by the constant $\delta_{1}$.

In this analysis, we will use several triangulations. The mesh $\mathscr{T}$ will be the globally refined, shape regular, quasiuniform, and conforming mesh of size $h$. We assume that the fine mesh $\mathscr{T}$ is aligned with the interface system $\Gamma$. There is a coarse mesh $\mathscr{T}^{0}$, also shape regular, conforming, and aligned with the interface system $\Gamma$. In the interior parts of the subdomains $\Omega_{i}, 1 \leq i \leq p$, the triangulation $\mathscr{T}^{0}$ is quasiuniform with elements of size $H \gg h$. To accommo- date the variant paradigm, near the interface system $\Gamma$ the mesh can be more refined. In particular, we will consider as a special case the situation where the fine interface system is completely represented in the mesh $\mathscr{T}^{0}$. To maintain shape regularity, the mesh $\mathscr{T}^{0}$ is graded in an appropriate (shape regular) fashion from the more refined elements near the interface $\Gamma$ to the coarse elements of size $H$ in the interiors of the $\Omega_{i}$.

The triangulations $\mathscr{T}^{i}, 1 \leq i \leq p$ are partially refined triangulations; they coincide with the fine triangulation $\mathscr{T}$ within $\Omega_{i}$, but largely coincide with the coarse triangulation $\mathscr{T}^{0}$ elsewhere. We assume that the triangulations are nested in the following sense: for $1 \leq i \leq p$, we have $\mathscr{T}^{0} \subset \mathscr{T}^{i} \subset$ $\mathscr{T}$. The special case where the complete interface system is represented in $\mathscr{T}^{0}$ is the most simple situation. In particular, $\mathscr{T}^{i}$ exactly coincides with $\mathscr{T}$ in $\Omega_{i}$, and exactly coincides with $\mathscr{T}^{0}$ in $\Omega_{j}, j \neq i$.

In the case that the interface system is not represented in $\mathscr{T}^{0}, \mathscr{T}^{i}$ is a nonuniform refinement of $\mathscr{T}^{0}$, where the refinement is mainly restricted to $\Omega_{i}$. Since the interface system is coarse, edges in $\Gamma \cap \partial \Omega_{i}$ are refined, requiring some additional (graded) refinements outside of $\Omega_{i}$ in order to maintain conformity and shape regularity in the mesh. Thus, given two triangulations, $\mathscr{T}^{i}$ and $\mathscr{T}^{j}$, a coarse subdomain $\Omega_{k}, k \neq i, k \neq j$, may be triangulated differently in the two cases, especially if $\Omega_{k}$ shares an interface with $\Omega_{i}$ or $\Omega_{j}$.

Let $\mathscr{S}$ denote the space of piecewise linear polynomials, associated with the triangulation $\mathscr{T}$, that are continuous in each of the $\Omega_{i}$, but can be discontinuous along the interface system $\Gamma$. Let $\overline{\mathscr{S}} \subset \mathscr{S}$ denote the subspace of globally continuous piecewise linear polynomials. The usual basis for $\mathscr{S}$ is just the union of the nodal basis functions corresponding to each of the subdomains $\Omega_{i}$; such basis functions have their support in $\bar{\Omega}_{i}$ and those associated with nodes on $\Gamma$ will have a jump at the interface. In the theory, we will have occasion to consider another basis, allowing us to write $\mathscr{S}=\overline{\mathscr{S}} \oplus \mathscr{X}$, where $\mathscr{X}$ is a subspace associated exclusively with jumps on $\Gamma$. In particular, we will use the global conforming nodal basis for the space $\overline{\mathscr{S}}$, and construct a basis for $\mathscr{X}$ as follows. Let $z_{k}$ be a vertex lying on $\Gamma$ shared by two regions $\Omega_{i}$ and $\Omega_{j}$ (for now, $z_{k}$ is not a crosspoint). Let $\phi_{i, k}$ and $\phi_{j, k}$ denote the usual nodal basis functions corresponding to $z_{k}$ in $\Omega_{i}$ and $\Omega_{j}$, respectively. The continuous nodal basis function for $z_{k}$ in $\overline{\mathscr{S}}$ is $\phi_{k} \equiv \phi_{i, k}+\phi_{j, k}$, and the "jump" basis function in $\mathscr{X}$ is $\hat{\phi}_{k} \equiv \phi_{i, k}-\phi_{j, k}$. The direction of the jump is arbitrary at each $z_{k}$, but once chosen, will be used consistently. In this example, at point $z_{k}$ we will refer to $i$ and the "master" index and $j$ as the "slave" index. At a cross point where $\ell>2$ subregions meet, there will be one nodal basis function corresponding to $\overline{\mathscr{S}}$ and $\ell-1$ jump basis functions. These are constructed by choosing one master index for the point, and making the other $\ell-1$ indices slaves. We can construct $\ell-1$ basis functions for $\mathscr{X}$ as $\phi_{i, k}-\phi_{j, k}$, where $i$ is the master index and $j$ is one of the slave indices.

For each of the triangulations $\mathscr{T}^{i}, 1 \leq i \leq p$, and for the global coarse triangulation $\mathscr{T}^{0}$, we have a global nonconforming subspace $\mathscr{S}^{i} \subset \mathscr{S}$, and global conforming subspace 
$\overline{\mathscr{S}}^{i} \subset \overline{\mathscr{S}}$. In a fashion similar to $\mathscr{S}$, we have $\mathscr{S}^{i}=\overline{\mathscr{S}}^{i} \oplus \mathscr{X}^{i}$ In the special case that $\mathscr{T}^{0}$ contains the globally refined interface system, $\mathscr{X}^{i} \equiv \mathscr{X}, 1 \leq i \leq p$.

Let the continuous variational problem be: find $u \in$ $\mathscr{H}^{1}(\Omega)$ such that

$a(u, v)=(f, v)$

for all $v \in \mathscr{H}^{1}(\Omega)$, where

$a(u, v)=\int_{\Omega} a \nabla u \cdot \nabla v+b u v d x$,

$(f, v)=\int_{\Omega} f v d x$

$\|u\|_{\Omega}^{2}=a(u, u)$.

We assume that $a>0, b \geq 0$ are smooth and chosen such that $a(\cdot, \cdot)$ is coercive, so that $\|\cdot\|_{\Omega}$ defines a strong norm on $\mathscr{H}^{1}(\Omega)$, comparable to the usual $\|\cdot\|_{1, \Omega}$. The case of a singular Neumann problem presents no difficulties; the usual compatibility condition

$(f, 1)=0$

applies, and the solution is made unique by requiring

$(u, 1)=0$.

To deal with the nonconforming nature of $\mathscr{S}$, for $u, v \in$ $\mathscr{S}$, we define

$$
\begin{aligned}
a(u, v) & =\sum_{i=1}^{p} \int_{\Omega_{i}} a \nabla u \cdot \nabla v+b u v d x \\
& =\sum_{i=1}^{p} a_{\Omega_{i}}(u, v)
\end{aligned}
$$

For each vertex $z$ lying on $\Gamma$ there is one master index and $\ell-1>0$ slave indices. The total number of slave indices is denoted by $K$, so the total number of constraint equations in our nonconforming method is $K$. To simplify notation, for each $1 \leq j \leq K$, let $m(j)$ denote the corresponding master index, and $z_{j}$ the corresponding vertex. We define the bilinear form $b(v, \lambda)$ by

$b(v, \lambda)=\sum_{j=1}^{K}\left\{v_{m(j)}-v_{j}\right\} \lambda_{j}$

where $\lambda \in \mathscr{R}^{K}$. In words, $b(\cdot, \cdot)$ measures the jump between the master value and each of the slave values at each vertex on $\Gamma$. The nonconforming variational formulation of (1) is: find $u_{h} \in \mathscr{S}$ such that

$a\left(u_{h}, v\right)+b(v, \lambda)=(f, v)$

$b\left(u_{h}, \xi\right) \quad=0$

for all $v \in \mathscr{S}$ and $\xi \in \mathscr{R}^{K}$. Although this is formally a saddle point problem, the constraints are very simple; in particular, (3) simply imposes continuity at each of the vertices lying on
$\Gamma$, which in turn, implies that $u_{h} \in \overline{\mathscr{S}}$. Thus $u_{h}$ also solves the reduced and conforming variational problem: find $u_{h} \in$ $\overline{\mathscr{S}}$ such that

$a\left(u_{h}, v\right)=(f, v)$

for all $v \in \overline{\mathscr{S}}$.

In the triangulations $\mathscr{T}^{i}$, the mesh near $\Gamma$ may not be as refined as in $\mathscr{T}$ in $\Omega_{j}, j \neq i$. Let $\mathscr{K}_{i}$ denote the index set of constraint equations in (2) that correspond to vertices present in $\mathscr{T}^{i}$. Then

$b_{i}(v, \lambda)=\sum_{j \in \mathscr{K}_{i}}\left\{v_{m(j)}-v_{j}\right\} \lambda_{j}$.

If the interface is completely refined $b_{i}(\cdot, \cdot) \equiv b(\cdot, \cdot)$.

We are now in a position to formulate our domain decomposition algorithm. We first consider the initial guess $u_{0} \in \mathscr{S}$, generated as follows: for $1 \leq i \leq p$, we find (in parallel) $u_{0, i} \in \overline{\mathscr{S}}^{i}$ satisfying

$a\left(u_{0, i}, v\right)=(f, v)$

for all $v \in \overline{\mathscr{S}}^{i}$. Note that here we assume exact solution of these local problems; in the actual implementation, these are solved approximately using the multigraph algorithm. The initial guess $u_{0} \in \mathscr{S}$ is composed by taking the part of $u_{0, i}$ corresponding to the fine subregion $\Omega_{i}$ for each $i$. In particular, let $\chi_{i}$ be the characteristic function for the subregion $\Omega_{i}$. Then

$u_{0}=\sum_{i=1}^{p} \chi_{i} u_{0, i}$

To compute $u_{k+1} \in \mathscr{S}$ from $u_{k} \in \mathscr{S}$, we solve (in parallel): for $1 \leq i \leq p$, find $e_{k, i} \in \mathscr{S}^{i}$ and $\lambda_{k, i} \in \mathscr{R}^{K}$ such that

$a\left(e_{k, i}, v\right)+b_{i}\left(v, \lambda_{k, i}\right)=(f, v)-a\left(u_{k}, v\right)$

$b_{i}\left(e_{k, i}, \xi\right) \quad=-b_{i}\left(u_{k}, \xi\right)$

for all $v \in \mathscr{S}^{i}$ and $\xi \in \mathscr{R}^{K}$. We then form

$e_{k}=\sum_{i=1}^{p} \chi_{i} e_{k, i}$,

$u_{k+1}=u_{k}+e_{k}$.

We pause to make a few remarks. First, although the $u_{k}$ and $e_{k}$ are elements of the nonconforming space $\mathscr{S}$, the limit function $u_{\infty}=u_{h}$ belongs to the conforming finite element space $\overline{\mathscr{S}}$. In some sense, the purpose of the iteration is to drive the jumps in the approximate solution $u_{k}$ to zero. Second, although (7) suggests a saddle point problem needs to be solved, by recognizing that only $\chi_{i} e_{k, i}$ is actually used, one can reduce (7) to a positive definite problem of the form (5). In particular, the Lagrange multipliers $\lambda_{k, i}$ need not be computed or updated. This aspect is described in detail in Section 5 . 
Let $\hat{e}_{k}=u_{h}-u_{k}$ denote the exact error in $u_{k}$. Then $\hat{e}_{k}$ satisfies the saddle point problem: find $\hat{e}_{k} \in \mathscr{S}$ and $\lambda_{k} \in \mathscr{R}^{K}$ such that

$$
\begin{aligned}
& a\left(\hat{e}_{k}, v\right)+b\left(v, \lambda_{k}\right)=(f, v)-a\left(u_{k}, v\right) \\
& b\left(\hat{e}_{k}, \xi\right) \quad=-b\left(u_{k}, \xi\right)
\end{aligned}
$$

for all $v \in \mathscr{S}$ and $\xi \in \mathscr{R}^{K}$.

By comparing (7) and (9), we see that

$$
\begin{array}{ll}
a\left(\hat{e}_{k}-e_{k, i}, v\right)+b_{i}\left(v, \lambda_{k}-\lambda_{k, i}\right) & =0 \\
b_{i}\left(\hat{e}_{k}-e_{k, i}, \xi\right) & =0
\end{array}
$$

for all $v \in \mathscr{S}^{i}$ and $\xi \in \mathscr{R}^{K}$. For the special case where $b_{i}(\cdot, \cdot) \equiv b(\cdot, \cdot)$ we also have the more simple projection-like relation

$a\left(\hat{e}_{k}-e_{k, i}, v\right)=0$

for all $v \in \overline{\mathscr{S}}^{i}$. From the identity

$v=\sum_{i=1}^{p} \chi_{i} v$

for all $v \in \mathscr{S}$, we have

$\hat{e}_{k+1}=\hat{e}_{k}-e_{k}=\sum_{i=1}^{p} \chi_{i}\left(\hat{e}_{k}-e_{k, i}\right)$.

\section{Derivation of the Error Propagator}

In this section we will derive, in matrix/operator notation, the error propagator for the iteration described in Section 2. We will begin for the simple case of $p=2$ subregions. We then generalize to the case of general $p$, but with a completely refined interface system on each processor (i.e., $\left.b_{i}(\cdot, \cdot) \equiv b(\cdot, \cdot)\right)$. Finally we consider general $p$, with a coarse interface system in the region outside of a given processors' subdomain.

\subsection{The case $p=2$}

In the case $p=2$, the global matrix for the saddle point problem is given by

$A=\left(\begin{array}{ccc}A_{1} & 0 & B_{1}^{t} \\ 0 & A_{2} & B_{2}^{t} \\ B_{1} & B_{2} & 0\end{array}\right)$.

The matrices $A_{i}$ correspond to the bilinear forms $a_{\Omega_{i}}(\cdot, \cdot)$ relative to the global fine mesh $\mathscr{T}$. The matrices $B_{i}$ correspond to the bilinear form $b(\cdot, \cdot)$; these matrices are rectangular with one nonzero entry $( \pm 1)$ for each row that corresponds to a constraint equation on $\partial \Omega_{i}$; this is all rows for the case $p=2$, but in general many rows of $B_{i}$ will contain all zeroes. The global Schur complement is given by

$S=B_{1} A_{1}^{-1} B_{1}^{t}+B_{2} A_{2}^{-1} B_{2}^{t}$.
We pause here to note that some $A_{i}$ may be singular. In particular, although the bilinear form (1) is coercive, any given $A_{i}$ could have a one dimensional kernel corresponding to the constant function. However, each column of $B_{i}^{t}$ forms a consistent right hand side so that (14) remains well defined if we replace $A_{i}^{-1}$ by its generalized inverse, and is the correct limit if we perturb $A_{i}$ slightly such that it is positive definite, and then study the limiting behavior of $S$. Similar remarks apply to all the cases later in this section when similar situations could arise; thus in the remainder of this section, matrix inverses will be understood to be generalized inverses as necessary.

The restriction operator mapping $\mathscr{S} \rightarrow \mathscr{S}^{1}$ is:

$Q_{1}=\left(\begin{array}{ccc}I & 0 & 0 \\ 0 & P_{2}^{t} & 0 \\ 0 & 0 & I\end{array}\right)$

The matrix $P_{2}^{t}$ is rectangular, and contains coefficients that express coarse grid basis functions for region two as linear combinations of fine grid basis functions. It is similar to the Galerkin restriction and prolongation matrices commonly employed in multigrid analysis. The partition of unity matrix for region one is

$\chi_{1}=\left(\begin{array}{lll}I & 0 & 0 \\ 0 & 0 & 0 \\ 0 & 0 & 0\end{array}\right)$.

Let

$\bar{A}_{2}=P_{2}^{t} A_{2} P_{2}$

$\bar{B}_{2}=B_{2} P_{2}$

$\pi_{2}=I-P_{2} \bar{A}_{2}^{-1} P_{2}^{t} A_{2}$

$S_{1}=B_{1} A_{1}^{-1} B_{1}^{t}+\bar{B}_{2} \bar{A}_{2}^{-1} \bar{B}_{2}^{t}$

$=B_{1} A_{1}^{-1} B_{1}^{t}+B_{2}\left(I-\pi_{2}\right) A_{2}^{-1} B_{2}^{t}$

$E_{1}=A_{1}^{-1} B_{1}^{t} S_{1}^{-1}$.

Note that the elliptic projection $\pi_{2}$, similar to a multigrid coarse grid correction, removes low frequency components from region two, whereas the extension operator $E_{1}$ makes smooth extension of the data restricted to the interface into region one.

The subdomain solver for region one is

$$
\begin{aligned}
& M_{1}=Q_{1} A Q_{1}^{t}=\left(\begin{array}{ccc}
A_{1} & 0 & B_{1}^{t} \\
0 & \bar{A}_{2} & \bar{B}_{2}^{t} \\
B_{1} & \bar{B}_{2} & 0
\end{array}\right)
\end{aligned}
$$

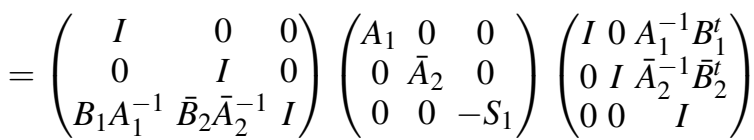

Using the factorization, it is easy to compute

$\chi_{1} M_{1}^{-1} Q_{1} A=\left(\begin{array}{ccc}I & E_{1} B_{2} \pi_{2} & 0 \\ 0 & 0 & 0 \\ 0 & 0 & 0\end{array}\right)$ 
A similar calculation holds for region two. Let

$$
\begin{aligned}
& Q_{2}=\left(\begin{array}{lll}
P_{1}^{t} & 0 & 0 \\
0 & I & 0 \\
0 & 0 & I
\end{array}\right) \\
& \chi_{2}=\left(\begin{array}{lll}
0 & 0 & 0 \\
0 & I & 0 \\
0 & 0 & 0
\end{array}\right)
\end{aligned}
$$$$
M_{2}=Q_{2} A Q_{2}^{t}=\left(\begin{array}{ccc}
\bar{A}_{1} & 0 & \bar{B}_{1}^{t} \\
0 & A_{2} & B_{2}^{t} \\
\bar{B}_{1} & B_{2} & 0
\end{array}\right)
$$

$$
\bar{A}_{1}=P_{1}^{t} A_{1} P_{1}
$$$$
\bar{B}_{1}=B_{1} P_{1}
$$$$
\pi_{1}=I-P_{1} \bar{A}_{1}^{-1} P_{1}^{t} A_{1}
$$$$
S_{2}=\bar{B}_{1} \bar{A}_{1}^{-1} \bar{B}_{1}^{t}+B_{2} A_{2}^{-1} B_{2}^{t}
$$$$
=B_{1}\left(I-\pi_{1}\right) A_{1}^{-1} B_{1}^{t}+B_{2} A_{2}^{-1} B_{2}^{t}
$$

$E_{2}=A_{2}^{-1} B_{2}^{t} S_{2}^{-1}$.

Similar to region one, we have

$$
\chi_{2} M_{2}^{-1} Q_{2} A=\left(\begin{array}{ccc}
0 & 0 & 0 \\
E_{2} B_{1} \pi_{1} & I & 0 \\
0 & 0 & 0
\end{array}\right)
$$

Using (17)-(18), the global error propagator is given by

$$
\begin{aligned}
G & =I-\chi_{1} M_{1}^{-1} Q_{1} A-\chi_{2} M_{2}^{-1} Q_{2} A \\
& =-\left(\begin{array}{ccc}
0 & E_{1} B_{2} \pi_{2} & 0 \\
E_{2} B_{1} \pi_{1} & 0 & 0 \\
0 & 0 & -I
\end{array}\right) .
\end{aligned}
$$

The $I$ in the $(3,3)$ block arises because we do not compute or update the Lagrange multipliers. The fact that the block third row and column are otherwise zero shows that failing to compute Lagrange multipliers does not affect the error in the other components of the solution. Thus, since we are only interested in the error in the solution itself, it suffices to consider on the block $2 \times 2$ error propagator

$\bar{G}=-\left(\begin{array}{cc}0 & E_{1} B_{2} \pi_{2} \\ E_{2} B_{1} \pi_{1} & 0\end{array}\right)$.

We note that $\bar{G}$ is not a symmetric operator (including the energy norm).

\subsection{The case of general $p$}

We now consider the case of general $p$, assuming that the global interface system is completely represented on all processors. In analogy with (13), the global matrix for the saddle point problem is given by

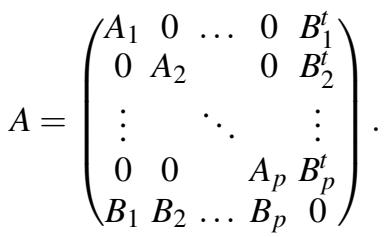

The global Schur complement is given by

$S=\sum_{j=1}^{p} B_{j} A_{j}^{-1} B_{j}^{t}$.

We will derive the contribution to the global error propagator associated with region one. The remaining regions follow a similar pattern. In this context, it is useful to consider regions $2-p$ as a single block and express the global matrix as

$A=\left(\begin{array}{ccc}A_{1} & 0 & B_{1}^{t} \\ 0 & A_{*} & B_{*}^{t} \\ B_{1} & B_{*} & 0\end{array}\right)$.

Note $A_{*}$ is a block diagonal matrix, and $B_{*}^{t}$ is a block vector. We can now follow the derivation for the case $p=2$; in analogy with (15), the restriction operator for region one is:

$Q_{1}=\left(\begin{array}{ccc}I & 0 & 0 \\ 0 & P_{*}^{t} & 0 \\ 0 & 0 & I\end{array}\right)$,

where $P_{*}$ is a block diagonal matrix. The partition of unity matrix for region one is given by (16). The subdomain solver for region one is

$$
\begin{aligned}
M_{1} & =Q_{1} A Q_{1}^{t} \\
& =\left(\begin{array}{ccc}
I & 0 & 0 \\
0 & I & 0 \\
B_{1} A_{1}^{-1} & \bar{B}_{*} \bar{A}_{*}^{-1} & I
\end{array}\right)\left(\begin{array}{ccc}
A_{1} & 0 & 0 \\
0 & \bar{A}_{*} & 0 \\
0 & 0 & -S_{1}
\end{array}\right)\left(\begin{array}{lll}
I & 0 & A_{1}^{-1} B_{1}^{t} \\
0 & I & \bar{A}_{*}^{-1} \bar{B}_{*}^{t} \\
0 & 0 & I
\end{array}\right)
\end{aligned}
$$

where

$\bar{A}_{*}=P_{*}^{t} A_{*} P_{*}$

$\bar{B}_{*}=B_{*} P_{*}$

$\pi_{*}=I-P_{*} \bar{A}_{*}^{-1} P_{*}^{t} A_{*}$

$S_{1}=B_{1} A_{1}^{-1} B_{1}^{t}+\bar{B}_{*} \bar{A}_{*}^{-1} \bar{B}_{*}^{t}$

$$
=B_{1} A_{1}^{-1} B_{1}^{t}+\sum_{j=2}^{p} \bar{B}_{j} \bar{A}_{j}^{-1} \bar{B}_{j}^{t}
$$

$E_{1}=A_{1}^{-1} B_{1}^{t} S_{1}^{-1}$.

Note in particular that $\pi_{*}$ is block diagonal. Using the factorization, and following the pattern for the case $p=2$ it is easy to compute

$\chi_{1} M_{1}^{-1} Q_{1} A=\left(\begin{array}{ccc}I & E_{1} B_{*} \pi_{*} & 0 \\ 0 & 0 & 0 \\ 0 & 0 & 0\end{array}\right)$.

We can express this using the expanded block structure as

$\chi_{1} M_{1}^{-1} Q_{1} A=\left(\begin{array}{cccccc}I & E_{1} B_{2} \pi_{2} & \ldots & E_{1} B_{p} \pi_{p} & 0 \\ 0 & 0 & \ldots & 0 & 0 \\ \vdots & & & & \vdots \\ 0 & 0 & \ldots & 0 & 0\end{array}\right)$ 
After making a similar calculation for the remaining regions, we can compute the global error propagator given by

$$
\begin{aligned}
G & =I-\sum_{j=1}^{p} \chi_{j} M_{j}^{-1} Q_{j} A \\
& =-\left(\begin{array}{ccccc}
0 & E_{1} B_{2} \pi_{2} & \ldots & E_{1} B_{p} \pi_{p} & 0 \\
E_{2} B_{1} \pi_{1} & 0 & & E_{2} B_{p} \pi_{p} & 0 \\
\vdots & & \ddots & & \vdots \\
E_{p} B_{1} \pi_{1} & E_{p} B_{2} \pi_{2} & & 0 & 0 \\
0 & 0 & \ldots & 0 & -I
\end{array}\right) .
\end{aligned}
$$

As before the appearance of the identity in the last row corresponds to the fact that we do not compute the Lagrange multipliers as part of the iteration. As in the case $p=2$, we can restrict attention to the block $p \times p$ matrix

$\bar{G}=-\left(\begin{array}{cccc}0 & E_{1} B_{2} \pi_{2} & \ldots & E_{1} B_{p} \pi_{p} \\ E_{2} B_{1} \pi_{1} & 0 & & E_{2} B_{p} \pi_{p} \\ \vdots & & \ddots & \\ E_{p} B_{1} \pi_{1} & E_{p} B_{2} \pi_{2} & & 0\end{array}\right)$

We note that $\bar{G}$ can be factored as

$\bar{G}=-\bar{E} \overline{\mathscr{J}} \bar{\pi}$

where

$\bar{E}=\left(\begin{array}{cccc}E_{1} & & & \\ & E_{2} & & \\ & & \ddots & \\ & & & E_{p}\end{array}\right)$,

$\bar{\pi}=\left(\begin{array}{llll}B_{1} \pi_{1} & & & \\ & B_{2} \pi_{2} & & \\ & & \ddots & \\ & & & B_{p} \pi_{p}\end{array}\right)$,

$\overline{\mathscr{J}}=\left(\begin{array}{cccc}0 & \mathscr{J}_{2} & \ldots & \mathscr{J}_{p} \\ \mathscr{J}_{1} & 0 & & \mathscr{J}_{p} \\ \vdots & & \ddots & \\ \mathscr{J}_{1} & \ldots & \mathscr{J}_{p-1} & 0\end{array}\right)$.

Here $\mathscr{J}_{i}$ is a diagonal matrix with zeros and ones on the diagonal. A diagonal entry is one if the corresponding constraint equation involves a point on the interface of region $i$, and is zero otherwise. In particular, note that $\mathscr{J}_{i} B_{i}=B_{i}$.

Let

$F_{i}=B_{i} \pi_{i} A_{i}^{-1} B_{i}^{t}$

$S_{0}=\sum_{j=1}^{p} \bar{B}_{j} \bar{A}_{j}^{-1} \bar{B}_{j}^{t}=S_{i}-F_{i}$

$\hat{F}_{i}=S_{0}^{-1 / 2} F_{i} S_{0}^{-1 / 2}$

for $1 \leq i \leq p$. Then

$B_{i} \pi_{i} A_{i}^{-1} B_{i}^{t} S_{i}^{-1}=F_{i}\left(S_{0}+F_{i}\right)^{-1}=S_{0}^{1 / 2} \hat{F}_{i}\left(I+\hat{F}_{i}\right)^{-1} S_{0}^{-1 / 2}$
We note that $S_{0}$, the Schur complement for the coarse space $\mathscr{S}^{0}$, is symmetric and positive definite (taking into account possible constraints for the case of a singular Neumann problem), and $\hat{F}_{i}\left(I+\hat{F}_{i}\right)^{-1}$ is symmetric and positive semidefinite.

Let

$D=\operatorname{diag}\left(\mathscr{J}_{i}\right)$,

$V^{t}=\left(\begin{array}{llll}\mathscr{J}_{1} & \mathscr{J}_{2} & \ldots & \mathscr{J}_{p}\end{array}\right)$,

$W^{t}=(I I \ldots I)$.

Then

$\overline{\mathscr{J}}=W V^{t}-D$.

We also note that (since $\left.\mathscr{J}_{i} B_{i}=B_{i}\right)$

$\bar{G}=-\bar{E} \bar{J} \bar{\pi}=-\bar{E}\left(W W^{t}-I\right) \bar{\pi}$.

Let

$T=\operatorname{diag}\left(\left\{\hat{F}_{i}\left(I+\hat{F}_{i}\right)^{-1}\right\}^{1 / 2}\right)$.

Then

$-\left(W W^{t}-I\right) \bar{\pi} \bar{E}=-\operatorname{diag}\left(S_{0}^{1 / 2}\right)\left(W W^{t}-I\right) T^{2} \operatorname{diag}\left(S_{0}^{-1 / 2}\right)$.

Thus, in order to determine the asymptotic rate of convergence, the analysis of the error propagator $\bar{G}$ can be reduced to analyzing the symmetric error propagator

$\hat{G}=-T\left(W W^{t}-I\right) T$

\subsection{The case of general $p$ with coarsened interface}

We now assume that the global interface system is coarsened on each processor. Our goal is to determined the changes relative to the case of the globally refined interface system considered in the last subsection. Rather than make a complete derivation, we will concentrate on the differences. As before, we will consider just the contribution to the global error propagator due to processor one.

Generalizing (21), the restriction operator for region one now is defined by:

$Q_{1}=\left(\begin{array}{ccc}I & 0 & 0 \\ 0 & P_{*}^{t} & 0 \\ 0 & 0 & R_{1}\end{array}\right)$,

where $P_{*}$ is a block diagonal matrix as before, and $R_{1}$ is a restriction matrix for the Lagrange multipliers. $R_{1}$ is rectangular; each row has a single entry of 1 and the remaining entries are 0 ; essentially $R_{1}$ selects the constraint equations to be imposed on processor one; note that this is the set of all constraint equations for interface grid points present in $\mathscr{T}^{1}$, and in particular, all constraint equations for the interface boundary of $\Omega_{1}$ in $\mathscr{T}$. 
The partition of unity matrix for region one is still given by (16). Let

$\bar{S}_{1}=R_{1} S_{1} R_{1}^{t}$

$C_{1}=I-R_{1}^{t} \bar{S}_{1}^{-1} R_{1} S_{1}$

$\bar{E}_{1}=A_{1}^{-1} B_{1}^{t} R_{1}^{t} \bar{S}_{1}^{-1} R_{1}$.

$S_{1}$ is the Schur complement, defined previously, obtained if all the interface constraints are imposed on processor one; because of the structure of $R_{1}, \bar{S}_{1}$ is a submatrix of $S_{1}$ corresponding to the constraint equations which are imposed on processor one. $C_{1}$ is the corresponding projection matrix. Note that now $\bar{E}_{1}$ is defined using the restricted Schur complement; if all constraints are imposed, $R_{1}=I$, and $\bar{E}_{1}=E_{1}$.

Following the pattern of the previous subsections, it is straightforward to see, in analogy to (24),

$\chi_{1} M_{1}^{-1} Q_{1} A=\left(\begin{array}{ccccc}I & \bar{E}_{1} B_{2} \pi_{12} & \ldots & \bar{E}_{1} B_{p} \pi_{1 p} A_{1}^{-1} B_{1}^{t} C_{1} \\ 0 & 0 & \ldots & 0 & 0 \\ \vdots & & & & \vdots \\ 0 & 0 & \ldots & 0 & 0\end{array}\right)$

The global error propagator is given by

$$
\begin{aligned}
G & =I-\sum_{j=1}^{p} \chi_{j} M_{j}^{-1} Q_{j} A \\
& =-\left(\begin{array}{ccccc}
0 & \bar{E}_{1} B_{2} \pi_{12} & \ldots & \bar{E}_{1} B_{p} \pi_{1 p} & A_{1}^{-1} B_{1}^{t} C_{1} \\
\bar{E}_{2} B_{1} \pi_{21} & 0 & & \bar{E}_{2} B_{p} \pi_{2 p} & A_{2}^{-1} B_{2}^{t} C_{2} \\
\vdots & & \ddots & & \vdots \\
\bar{E}_{p} B_{1} \pi_{p 1} & \bar{E}_{p} B_{2} \pi_{p 2} & & 0 & A_{p}^{-1} B_{p}^{t} C_{p} \\
0 & 0 & \ldots & 0 & -I
\end{array}\right) .
\end{aligned}
$$

As before the appearance of the identity in the last row corresponds to the fact that we do not compute the Lagrange multipliers as part of the iteration. However, the last block column of $G$ is not otherwise zero as in the previous case (25). Thus the fact that not all constraints are imposed on all processors does influence the convergence of the overall iteration. However, because $G$ remains block upper triangular, this block column does not effect the eigenvalues of the iteration matrix and hence is asymptotically benign. So, as in the previous cases, we can restrict attention to the block $p \times p$ matrix

$\bar{G}=-\left(\begin{array}{cccc}0 & \bar{E}_{1} B_{2} \pi_{12} & \ldots & \bar{E}_{1} B_{p} \pi_{1 p} \\ \bar{E}_{2} B_{1} \pi_{21} & 0 & & \bar{E}_{2} B_{p} \pi_{2 p} \\ \vdots & & \ddots & \\ \bar{E}_{p} B_{1} \pi_{p 1} & \bar{E}_{p} B_{2} \pi_{p 2} & & 0\end{array}\right)$

A more serious problem arises in the local projectors, now denoted $\pi_{i j}$. The extra subscript is needed because, e.g., the coarse triangulation of $\Omega_{2}$ on processor one may now be different from the coarse triangulation of $\Omega_{2}$ on processor three; hence $\pi_{12} \neq \pi_{32}$.
Because of this, $\bar{G}$ can no longer be factored as in (27). However, we still have the factorization

$\bar{G}=-\bar{E} \bar{\Pi}$

where

$\begin{aligned} \bar{E} & =\left(\begin{array}{cccc}\bar{E}_{1} & & & \\ & \bar{E}_{2} & & \\ & & \ddots & \\ & & & \bar{E}_{p}\end{array}\right), \\ \bar{\Pi} & =\left(\begin{array}{cccc}0 & B_{2} \pi_{12} & \ldots & B_{p} \pi_{1 p} \\ B_{1} \pi_{21} & 0 & & B_{p} \pi_{2 p} \\ \vdots & & \ddots & \\ B_{1} \pi_{p 1} & \ldots & B_{p-1} \pi_{p p-1} & 0\end{array}\right) .\end{aligned}$

\section{Convergence Analysis for a Special Case}

Our goal is to analyze the asymptotic behavior of the error propagator $\bar{G}=-\bar{E} \bar{J} \bar{\pi}$ in (27). Since $\bar{G}^{k}$ is the relevant operator for $k$ iterations, it is sufficient to consider the operator $\bar{\pi} \bar{E} \bar{J}$. Notice that $\bar{\pi} \bar{E}$ is a block diagonal (local) operator. In particular, the $i$-th diagonal block of $\bar{\pi} \bar{E}, B_{i} \pi_{i} A_{i}^{-1} B_{i}^{t} S_{i}^{-1}$, behaves as follows. We begin with some function values (errors) defined on the global interface system. $B_{i}^{t} S_{i}^{-1}$ takes this global system of interface errors and maps it into (discrete) Neumann data for subregion $i$, which is then extended (discrete harmonic) by $A_{i}^{-1}$ to all of $\bar{\Omega}_{i}$. We then apply the coarse grid projection $\pi_{i}$ to this smooth extension; this is very much like a multigrid coarse grid correction, and its effect on the smooth error is quite similar. The remaining (non-smooth) error after the projection is then restricted to the interface by $B_{i}$. The "mixing" matrix $\overline{\mathscr{J}}$ represents the global part of the iteration; $\overline{\mathscr{J}}$ takes the interface errors from region $i$ and broadcasts them to all other processors; region $i$ in turn receives similar errors from all other processors, and the local part of the cycle is repeated. Below we treat this process in the special case of a fully refined interface by analyzing the symmetric error propagator $\hat{G}$ of (29).

In what follows all estimates we derive are local and depend on the particular subdomain $\Omega_{i}$. In particular, we assume that $\Omega_{i}$ stay geometrically similar to a fixed number of reference polygons. Therefore, we can essentially assume without loss of generality that all subdomains are of unit size. This makes the relations between the various parameters of the method more transparent.

Let $\Omega_{i}$ be one of the domains. Let $\mathscr{S}_{h} \equiv \mathscr{S}_{h}\left(\Omega_{i}\right)$ denote the fine subspace on $\Omega_{i}$ (equivalently, the restriction of any of the global spaces $\mathscr{S}, \overline{\mathscr{S}}, \mathscr{S}^{i}$, or $\overline{\mathscr{S}}^{i}$ to $\Omega_{i}$ ). Let $\mathscr{S}_{H} \equiv \mathscr{S}_{H}\left(\Omega_{i}\right) \subset \mathscr{S}_{h}$ denote the partially coarsened space (restriction of the global spaces $\mathscr{S}^{0}, \overline{\mathscr{S}}^{0}, \mathscr{S}^{j}$, or $\overline{\mathscr{S}}^{j}, j \neq i$, to $\Omega_{i}$ ).

We consider three Neumann problems: First: find $u \in$ $\mathscr{H}^{1}\left(\Omega_{i}\right)$ such that

$a_{\Omega_{i}}(u, v)=\langle g, v\rangle_{\partial \Omega_{i}}$ 
for all $v \in \mathscr{H}^{1}\left(\Omega_{i}\right)$. Second: find $u_{h} \in \mathscr{S}_{h}$ such that

$a_{\Omega_{i}}\left(u_{h}, v\right)=\langle g, v\rangle_{\partial \Omega_{i}}$

for all $v \in \mathscr{S}_{h}$. Third: find $u_{H} \in \mathscr{S}_{H}$ such that

$a_{\Omega_{i}}\left(u_{H}, v\right)=\langle g, v\rangle_{\partial \Omega_{i}}$

for all $v \in \mathscr{S}_{H}$. The Neumann data $g$ is a finite element function from $\mathscr{S}_{h}$ (or equivalently, $\mathscr{S}_{H}$ ) restricted to $\partial \Omega_{i}$. We assume unique solutions to these problems, with the usual caveats of consistency $\left(\langle g, 1\rangle_{\partial \Omega_{i}}=0\right)$ and unique generalized solutions $\left((u, 1)_{\Omega_{i}}=\left(u_{h}, 1\right)_{\Omega_{i}}=\left(u_{H}, 1\right)_{\Omega_{i}}=0\right)$ for singular Neumann problems.

Since $\mathscr{S}_{H} \subset \mathscr{S}_{h} \subset \mathscr{H}^{1}\left(\Omega_{i}\right)$, we have the usual orthogonality relations

$$
\begin{aligned}
a_{\Omega_{i}}\left(u-u_{h}, v\right)=0 & \text { for all } v \in \mathscr{S}_{h}, \\
a_{\Omega_{i}}\left(u-u_{H}, v\right)=0 & \text { for all } v \in \mathscr{S}_{H}, \\
a_{\Omega_{i}}\left(u_{h}-u_{H}, v\right)=0 & \text { for all } v \in \mathscr{S}_{H} .
\end{aligned}
$$

From (38), it follows that

$$
\begin{gathered}
\left\|u-u_{h}\right\|_{\Omega_{i}}^{2}+\left\|u_{h}\right\|_{\Omega_{i}}^{2}=\|u\|_{\Omega_{i}}^{2}, \\
\left\|u-u_{H}\right\|_{\Omega_{i}}^{2}+\left\|u_{H}\right\|_{\Omega_{i}}^{2}=\|u\|_{\Omega_{i}}^{2}, \\
\left\|u_{h}-u_{H}\right\|_{\Omega_{i}}^{2}+\left\|u_{H}\right\|_{\Omega_{i}}^{2}=\|\| u_{h} \|_{\Omega_{i}}^{2} .
\end{gathered}
$$

From (39), we have

$$
\left\|u_{h}-u_{H}\right\|_{\Omega_{i}}^{2}+\left\|u-u_{h}\right\|_{\Omega_{i}}^{2}=\left\|u-u_{H}\right\|_{\Omega_{i}}^{2}
$$

and

$\left\|u_{H}\left|\left\|_{\Omega_{i}} \leq\right\|\left\|u_{h} \mid\right\| \Omega_{\Omega_{i}} \leq\|u\| \|_{\Omega_{i}}\right.\right.$.

\subsection{A global error estimate}

Theorem 1 Let $\hat{G}=-T\left(W W^{t}-I\right) T$ as in (29). Assume that the solutions of the local Neumann problems above satisfy the a priori estimate

$$
\left\|u_{h}-u_{H}\left|\left\|_{\Omega_{i}} \leq \gamma \mid\right\| u_{H} \|_{\Omega_{i}}\right.\right.
$$

for some $\gamma<1$. Then

$$
\left\|T\left(W W^{t}-I\right) T\right\|_{\ell_{2}} \leq \gamma^{2}
$$

Proof We begin by noting that

$$
T\left(W W^{t}-I\right) T=(T W)(T W)^{t}-T^{2} .
$$

Thus

$\left\|(T W)(T W)^{t}-T^{2}\right\|_{\ell_{2}} \leq \max \left(\|T W\|_{\ell_{2}}^{2},\left\|T^{2}\right\|_{\ell_{2}}\right)$.

We will bound each of these terms separately.
We consider first the term $\left\|T^{2}\right\|_{\ell_{2}}$. Since $\hat{F}_{i}$ is symmetric, positive semi-definite,

$$
\begin{aligned}
\left\|\hat{F}_{i}\left(I+\hat{F}_{i}\right)^{-1}\right\|_{\ell_{2}} & =\max _{x} \frac{x^{t} \hat{F}_{i} x}{x^{t}\left(I+\hat{F}_{i}\right) x} \\
& =\max _{x} \frac{x^{t}\left(S_{0}^{-1 / 2} F_{i} S_{0}^{-1 / 2}\right) x}{x^{t}\left(I+S_{0}^{-1 / 2} F_{i} S_{0}^{-1 / 2}\right) x} \\
& =\max _{x} \frac{x^{t} F_{i} x}{x^{t}\left(S_{0}+F_{i}\right) x} \\
& =\max _{x} \frac{x^{t} B_{i} \pi_{i} A_{i}^{-1} B_{i}^{t} x}{x^{t}\left(B_{i} A_{i}^{-1} B_{i}^{t}+\sum_{j \neq i} \bar{B}_{j} \bar{A}_{j}^{-1} \bar{B}_{j}^{t}\right) x} .
\end{aligned}
$$

Let $y_{i}=A_{i}^{-1} B_{i}^{t} x$. Then

$x^{t} B_{i} A_{i}^{-1} B_{i}^{t} x=y_{i}^{t} A_{i} y_{i}=\left\|\chi_{h}\right\|_{\Omega_{i}}^{2}$

for the corresponding $\chi_{h} \in \prod_{k=1}^{p} \mathscr{S}_{h}\left(\Omega_{k}\right) \equiv \mathscr{S}$. Note that the global function $\chi_{h}$ is determined by solving local problems in each of the subdomains $\Omega_{k}, 1 \leq k \leq p$; these problems all have related Neumann data as specified from $B_{k}^{t} x$. Also

$x^{t} B_{i} \pi_{i} A_{i}^{-1} B_{i}^{t} x=y_{i}^{t} A_{i} \pi_{i} y_{i}=\left\|\chi_{h}-\chi_{H}\right\|_{\Omega_{i}}^{2}$

where $\chi_{H} \in \prod_{k=1}^{p} \mathscr{S}_{H}\left(\Omega_{k}\right) \equiv \mathscr{S}^{0}$ is the (piecewise elliptic) projection of $\chi_{h}$ into $\mathscr{S}^{0}$. As with $\chi_{h}$, the projection $\chi_{H}$ is computed locally in each subdomain. Now let $y_{j}=\bar{A}_{j}^{-1} \bar{B}_{j}^{t} x$ for $j \neq i$. Then

$x^{t} \bar{B}_{j} \bar{A}_{j}^{-1} \bar{B}_{j}^{t} x=y_{j}^{t} \bar{A}_{j} y_{j}=\left\|\chi_{H}\right\|_{\Omega_{j}}^{2}$.

Returning now to (44), using (41) and (42)

$$
\begin{aligned}
\left\|\hat{F}_{i}\left(I+\hat{F}_{i}\right)^{-1}\right\|_{\ell_{2}} & =\max _{\chi_{h}} \frac{\left\|\chi_{h}-\chi_{H}\right\|_{\Omega_{i}}^{2}}{\left\|\chi_{h}\right\|\left\|_{\Omega_{i}}^{2}+\sum_{j \neq i}\right\| \chi_{H} \|_{\Omega_{j}}^{2}} \\
& \leq \max _{\chi_{h}} \frac{\left\|\chi_{h}-\chi_{H}\right\|_{\Omega_{i}}^{2}}{\left\|\chi_{h}\right\|_{\Omega_{i}}^{2}} \\
& \leq \max _{\chi_{h}} \frac{\left\|\chi_{h}-\chi_{H}\right\|_{\Omega_{i}}^{2}}{\left\|\chi_{H}\right\|_{\Omega_{i}}^{2}} \\
& \leq \gamma^{2} .
\end{aligned}
$$

Thus, based on the definition of $T$ and estimate (45) we have,

$\left\|T^{2}\right\|_{\ell_{2}}=\max _{i}\left\|\hat{F}_{i}\left(I+\hat{F}_{i}\right)^{-1}\right\|_{\ell_{2}} \leq \gamma^{2}$ 
We now turn to the term $\|T W\|_{\ell_{2}}$.

$$
\begin{aligned}
\|T W\|_{\ell_{2}}^{2} & =\sup _{x} \frac{x^{t} W^{t} T^{2} W x}{x^{t} x} \\
& =\sup _{x} \frac{\sum_{i=1}^{p} x^{t} \hat{F}_{i}\left(I+\hat{F}_{i}\right)^{-1} x}{x^{t} x} \\
& =\sup _{x} \frac{\sum_{i=1}^{p} x^{t} S_{0}^{\frac{1}{2}} \hat{F}_{i}\left(I+\hat{F}_{i}\right)^{-1} S_{0}^{\frac{1}{2}} x}{x^{t} S_{0} x} .
\end{aligned}
$$

Now use the fact that

$y^{t} \hat{F}_{i}\left(I+\hat{F}_{i}\right)^{-1} y \leq y^{t} \hat{F}_{i} y$,

which implies

$x^{t} S_{0}^{\frac{1}{2}} \hat{F}_{i}\left(I+\hat{F}_{i}\right)^{-1} S_{0}^{\frac{1}{2}} x \leq x^{t} S_{0}^{\frac{1}{2}} \hat{F}_{i} S_{0}^{\frac{1}{2}} x=x^{t} F_{i} x$.

Therefore, using (42),

$$
\begin{aligned}
\|T W\|_{\ell_{2}}^{2} & \leq \sup _{x} \sum_{i=1}^{p} \frac{x^{t} F_{i} x}{x^{t} S_{0} x} \\
& =\sup _{x} \sum_{i=1}^{p} \frac{\left(B_{i}^{t} x\right)^{t} \pi_{i} A_{i}^{-1} B_{i}^{t} x}{\sum_{j=1}^{p}\left(B_{j}^{t} x\right)^{t} \bar{A}_{j}^{-1} B_{j}^{t} x} \\
& =\sup _{\chi_{h}} \sum_{i=1}^{p} \frac{\left\|\chi_{h}-\chi_{H}\right\|_{\Omega_{i}}^{2}}{\sum_{j=1}^{p}\left\|\chi_{H}\right\|_{\Omega_{j}}^{2}} \\
& \leq \gamma^{2} .
\end{aligned}
$$

Thus we have

$$
\left\|(T W)(T W)^{t}-T^{2}\right\|_{\ell_{2}} \leq \max \left(\|T W\|_{\ell_{2}}^{2},\left\|T^{2}\right\|_{\ell_{2}}\right) \leq \gamma^{2}
$$

completing the proof.

\subsection{Some local error estimates}

We now make some local estimates for the solutions of (35)(37).

Theorem 2 Let $u, u_{h}$ and $u_{H}$ be defined as in (35)-(37). Then

$\left\|u_{H}\right\| \Omega_{\Omega_{i}} \leq\left\|u_{h}\right\|_{\Omega_{i}} \leq\|\| u\left\|_{\Omega_{i}} \leq C_{1}\right\| u_{H} \|_{\Omega_{i}}$

with $C_{1}$ independent of $p, N, h$, and $H$.
Proof The first two inequalities reprise (41). The right hand inequality in (46) holds because $g$ is the trace of a finite element function $\psi_{h} \in \mathscr{S}_{h}\left(\right.$ and $\left.\psi_{H} \in \mathscr{S}_{H}\right)$. Let $q_{H}: L_{2}\left(\partial \Omega_{i}\right) \mapsto$ $\left.\mathscr{S}_{H}\right|_{\partial \Omega_{i}}$ be the $L_{2}$-projection. Then, for any $\varphi \in H^{1}\left(\Omega_{i}\right)$ one has,

$a_{\Omega_{i}}(u, \varphi)=\langle g, \varphi\rangle_{\partial \Omega_{i}}=\left\langle g, q_{H} \varphi\right\rangle_{\partial \Omega_{i}}=a_{\Omega_{i}}\left(u_{H}, \widetilde{\left(q_{H} \varphi\right)}\right)$,

where $\widetilde{\left(q_{H} \varphi\right)} \in \mathscr{S}_{H}$ is any extension of $q_{H} \varphi$ to a function in $\mathscr{S}_{H}$ to the interior of $\Omega_{i}$. Therefore,

$$
\begin{aligned}
\|u\|_{\Omega_{i}} & =\sup _{\varphi \in H^{1}\left(\Omega_{i}\right)} \frac{a_{\Omega_{i}}\left(u_{H}, \widehat{\left(q_{H} \varphi\right)}\right)}{\|\varphi\|_{\Omega_{i}}} \\
& \leq\left\|u_{H}\right\| \|_{\Omega_{i}} \sup _{\varphi \in H^{1}\left(\Omega_{i}\right)} \frac{\left\|\widetilde{\left(q_{H} \varphi\right)}\right\| \Omega_{\Omega_{i}}}{\|\varphi\| \Omega_{\Omega_{i}}} .
\end{aligned}
$$

The extension $\widetilde{\left(q_{H} \varphi\right)}$ of the trace $q_{H} \varphi$ can be chosen so that it is bounded in energy, [29], i.e.,

$\left\|\widetilde{\left(q_{H} \varphi\right)}\right\|\left\|_{\Omega_{i}} \leq C\right\| q_{H} \varphi \|_{\frac{1}{2}, \partial \Omega_{i}}$.

By a trace inequality

$\|\varphi\|_{\frac{1}{2}, \partial \Omega_{i}} \leq C\|\varphi\|_{1, \Omega_{i}} \leq C\|\varphi\|_{\Omega_{i}}$.

Thus we obtain

\|\|$u\left\|\Omega_{\Omega_{i}} \leq C\right\| u_{H}\|\|_{\Omega_{i}} \sup _{\varphi \in H^{\frac{1}{2}}\left(\partial \Omega_{i}\right)} \frac{\left\|q_{H} \varphi\right\|_{\frac{1}{2}, \partial \Omega_{i}}}{\|\varphi\|_{\frac{1}{2}, \partial \Omega_{i}}}$.

The right inequality in (46) follows since the $L_{2}$-projection $q_{H}$ is bounded, i.e.,

$\left\|q_{H} \varphi\right\|_{\frac{1}{2}, \partial \Omega_{i}} \leq C\|\varphi\|_{\frac{1}{2}, \partial \Omega_{i}}$.

We now need to make a more careful characterization of the region of width $d$ near $\partial \Omega_{i} \backslash \partial \Omega$ where $\mathscr{S}_{h}$ and $\mathscr{S}_{H}$ exactly coincide. Let $\Omega_{d}$ denote the interior of $\Omega_{i}$ where the two spaces differ, and let $\Omega_{d} \subset \Omega_{\hat{d}} \subset \Omega_{i}$. Informally, $\partial \Omega_{\hat{d}}$ lies halfway between $\partial \Omega_{d}$ and $\partial \Omega_{i}$. More precisely, along $\partial \Omega_{i} \backslash \partial \Omega$, the distance from $\partial \Omega_{\hat{d}}$ to both $\partial \Omega_{d}$ and $\partial \Omega_{i}$ is of order $d / 2$. Finally, we assume that $d$ is sufficiently large (with respect to $H$ ), for example, we can assume $d \simeq m H$ for a sufficiently large integer $m$. Then, for a fixed $m$ we will have $d \simeq H$.

\section{Theorem 3 We have}

$\left\|u_{h}-u_{H}\right\|_{\Omega_{i}} \leq C_{1} C_{2} \frac{H}{d}\left\|u_{H}\right\|_{\Omega_{i}}$,

where $C_{1}$ is given in Theorem 2 and $C_{2}$ is independent of $p$, $N, d, h$, and $H$. 
Proof Let $\mathscr{I}_{H}$ denote an extension operator from $\mathscr{S}_{h}$ restricted to $\Omega_{i} \backslash \Omega_{d}$ into $\mathscr{S}_{H}\left(\Omega_{d}\right)$. We assume that $\mathscr{I}_{H}$ is bounded, i.e., that the following estimate holds,

$$
\left\|\left|\mathscr{I}_{H} v\right|\right\|_{\Omega_{d}} \leq C \inf _{w \in H^{1}\left(\Omega_{d}\right):\left.w\right|_{\Omega_{i} \backslash \Omega_{d}}=\left.v\right|_{\Omega_{i} \backslash \Omega_{d}}}\|w \mid\|_{\Omega_{d}} .
$$

The latter estimate represents the fact that $\mathscr{I}_{H}$ has a norm comparable with the minimum-norm continuous extension. For some explicit extension operators, see, for example [19]. In particular, it follows from (48) that

\|\| $\mathscr{I}_{H}\left(u_{h}-u_{H}\right)\left|\left\|\Omega_{\Omega_{d}} \leq C\left|\left\|u_{h}-u_{H} \mid\right\| \Omega_{\Omega_{d}}\right.\right.\right.$.

Using the fact that for all $v \in \mathscr{S}_{h}, v-\mathscr{I}_{H} v \equiv 0$ in $\Omega_{i} \backslash \Omega_{d}$, we have

$$
\begin{aligned}
\left\|u_{h}-u_{H}\right\|_{\Omega_{i}}^{2} & =a_{\Omega_{i}}\left(u_{h}-u_{H}, u_{h}-u_{H}\right) \\
& =a_{\Omega_{i}}\left(u_{h}-u_{H}, u_{h}-u_{H}-\mathscr{I}_{H}\left(u_{h}-u_{H}\right)\right) \\
& =a_{\Omega_{d}}\left(u_{h}-u_{H}, u_{h}-u_{H}-\mathscr{I}_{H}\left(u_{h}-u_{H}\right)\right)
\end{aligned}
$$

where $a_{\Omega_{d}}(\cdot, \cdot)$ denotes the restriction of $a_{\Omega_{i}}(\cdot, \cdot)$ to $\Omega_{d}$. Thus

$$
\left\|u_{h}-u_{H}\right\|_{\Omega_{i}} \leq C\left\{\left\|u_{h}-u_{H}\right\|\left\|_{\Omega_{d}}+\right\| \mathscr{I}_{H}\left(u_{h}-u_{H}\right)\|\|_{\Omega_{d}}\right\}
$$

Based on the boundedness of $\mathscr{I}_{H}$ one obtains

$$
\left\|u_{h}-u_{H}\left|\left\|_{\Omega_{i}} \leq C \mid\right\| u_{h}-u_{H} \|_{\Omega_{d}} .\right.\right.
$$

We now treat the right hand side of (49) using interior estimates. Let $\Omega_{d} \subset \Omega_{\hat{d}} \subset \Omega_{i}$ as described above. Then standard interior estimates for $\left\|u-u_{h}\right\|_{\Omega_{d}}[30,26]$ yield

$$
\left\|u-u_{h}\right\|_{\Omega_{d}} \leq C\left\{\|u-\chi\|_{\Omega_{\hat{d}}}+d^{-1}\left\|u-u_{h}\right\|_{0, \Omega_{i}}\right\}
$$

where $\chi \in \mathscr{S}_{h}$. The second term on the right hand side of (50) is handled by a standard duality estimate (AubinNitsche Lemma) [28, 15]

$$
\left\|u-u_{h}\right\|_{0, \Omega_{i}} \leq C h\left\|u-u_{h}\right\|_{\Omega_{i}} \leq C h\|u\|_{\Omega_{i}} .
$$

For the first term, we begin with the standard approximation estimate $[28,15]$

$$
\inf _{\chi \in \mathscr{S}_{h}}\|u-\chi\|_{\Omega_{\hat{d}}} \leq C h|u|_{2, \Omega_{\hat{d}}}
$$

We next use the interior regularity estimate for the harmonic function $u$ (c.f. [18]) to obtain

$$
|u|_{2, \Omega_{\hat{d}}} \leq C d^{-1}\|u\|_{1, \Omega_{i}}
$$

Combining these estimates, we finally have

$\left\|u-u_{h}\right\|_{\Omega_{d}} \leq C \frac{h}{d}\|u\|_{\Omega_{i}}$.

Applying this same approach to $\left\|u-u_{H}\right\|_{\Omega_{d}}$ yields the analogous estimate

$$
\left\|u-u_{H}\right\|_{\Omega_{d}} \leq C \frac{H}{d}\|u\|_{\Omega_{i}} .
$$

Finally, by the triangle inequality and Theorem 2

$$
\begin{aligned}
\left\|u_{h}-u_{H}\right\| \Omega_{\Omega_{d}} & \leq\left.\left\|u-u_{h}\right\|\right|_{\Omega_{d}}+\left\|u-u_{H}\right\| \Omega_{\Omega_{d}} \\
& \leq C_{2} \frac{H}{d}\|u\| \|_{\Omega_{i}} \\
& \leq C_{1} C_{2} \frac{H}{d}\left\|u_{H}\right\|_{\Omega_{i}} .
\end{aligned}
$$

We remark that in most interior estimates, be they finite element error estimates or interior regularity estimates, the constant $d$ is not tracked, but rather is included in the generic constant. However, in the current situation it is important to explicitly track $d$, since it is influenced to some extent by the important parameters $N, p, h$ and $H$. In our setting, we have assumed that the diameter of $\Omega_{i}$ is of unit size. In that case it is clear that all constants accumulated in the final one $C_{2}$ are independent of all parameters $N, p, h$ and $H$. Note that by changing variables we can transform the domain $\Omega_{i}$ into a domain $\widehat{\Omega}_{i}$ of unit size. The parameter $d$ then transforms to $\widehat{d}=\frac{d}{\operatorname{diam}\left(\Omega_{i}\right)}$. Applying estimate (50) for the unit size domain $\widehat{\Omega}_{i}$ with $d:=\widehat{d}=\frac{d}{\operatorname{diam}\left(\Omega_{i}\right)}$ and using change of variables back to the original domain $\Omega_{i}$, the estimate (50) is seen to hold. That is the constant $C$ in (50) stays independent of the various parameters $(N, p, h$ and $H)$. Similar arguments applies to the remaining estimates. Note that we have assumed that the subdomains $\Omega_{i}$ are geometrically similar to a fixed number of reference domains.

Using the estimate in Theorem 3, we can bound the rate of convergence $\gamma^{2}$ of Theorem 1 by

$$
\gamma^{2} \leq\left(C_{1} C_{2} \frac{H}{d}\right)^{2}
$$

We make a few remarks about estimate (51). As a theoretical (but not practical) point, if one were to fix $p$ and make $H$ sufficiently small that $H=h$ and $\mathscr{S}^{i} \equiv \mathscr{S}$ for $1 \leq i \leq p$, then $d \sim \min _{i} \operatorname{diam}\left(\Omega_{i}\right)$ and each processor would independently solve the global fine problem. In this special case, the actual convergence factor $\gamma^{2}=0$ since the method essentially becomes a direct method, although estimate (47) in Theorem 3 does not provide the obvious bound of zero when $u_{h} \equiv u_{H}$. However, since the constants $C_{1}$ and $C_{2}$ are independent of $H, h, d, N$, and $p$, one can, for example, fix $p, d$ and the desired rate of convergence $\gamma^{2}$ and choose $H \simeq \frac{d}{m}$ sufficiently small (or equivalently, $m \geq 1$ sufficiently large) such that

$$
\frac{d}{m} \simeq H \leq \frac{d \gamma}{C_{1} C_{2}}
$$

or

$m \geq \frac{C_{1} C_{2}}{\gamma}$

yielding direct control of the bound on the rate of convergence. 
On the other hand, the most common scenario in practice is that one solves a problem of fixed size on each processor, (typically the largest problem possible) and then increases the number of processors $p$ to increase the size of the global problem. As a practical matter, as $p$ increases the coarse mesh used for load balancing in the original paradigm should become finer. In the variant strategy, the coarse mesh revealed in Step II will also naturally become finer.

More specifically (under the assumption of $\Omega_{i} \subset \mathbb{R}^{D}$, ( $D=2$ or 3 ) being of unit size), the size $N_{i}$ of the $i$ th subproblem can be estimated (in the ideal case)

$N_{i}+(p-1)\left(d N_{i}+(1-d) H^{-D}\right) \simeq N_{i}+(p-1)\left(d N_{i}+H^{-D}\right)$.

Ideally, we want to keep this size of order $N_{i} \simeq h^{-D}$. This implies

$p-1<d^{-1}$

and also,

$h<\left(\frac{1}{p-1}\right)^{1 / D} H$.

The latter relation is satisfied for $h$ sufficiently small. Therefore, the main restriction is on $d$, which for large $p$ reads

$d<1 /(p-1)$

The convergence rate we proved is controlled by $m: m H=$ $d$. The restriction on the coarse mesh (for large p) hence reads

$m H=d<1 /(p-1)$.

The latter is a bit too restrictive in practice (for $p$ of order a few hundreds and higher). That is why we refer to this case as an ideal one. In practice, we should use subproblems with coarsened interfaces. In that case the size of the $i$ th subproblem reduces to

$$
\begin{aligned}
N_{i}+p_{0} d N_{i}+\left(p_{0}(1-d) H^{-D}+(p-\right. & \left.\left.p_{0}\right) H^{-D}\right) \simeq \\
& N_{i}+p_{0} d N_{i}+p H^{-D} .
\end{aligned}
$$

Here $p_{0}<p$ is a fixed number reflecting the number of neighbors (of $\Omega_{i}$ ) that have fine mesh near a strip of size $d$ around the interface boundary.

In either case, the natural relationship for this scenario is $d \sim H$. Thus we should have $H / d \sim$ constant (bounded by $\mathscr{O}\left(1 / p_{0}\right)$ as shown above), which by (51) corresponds to an observed rate of convergence independent of both $N$ and $p$.

\section{Implementation}

In this section we describe the implementation of the domain decomposition algorithm used to solve the global conforming linear systems arising in Step 3 of the Bank-Holst paradigm. Here we again use matrix notation, and note that the practical implementation differs in some respects from the idealized version of the algorithm described in Sections $2-4$. In this context, we consider the block $4 \times 4$ global saddle point problem given by

$\left(\begin{array}{cccc}A_{s s} & A_{s m} & A_{s i} & I \\ A_{m s} & A_{m m} & A_{m i} & -Z^{t} \\ A_{i s} & A_{i m} & A_{i i} & 0 \\ I & -Z & 0 & 0\end{array}\right)\left(\begin{array}{c}\delta U_{s} \\ \delta U_{m} \\ \delta U_{i} \\ \Lambda\end{array}\right)=\left(\begin{array}{c}R_{s} \\ R_{m} \\ R_{i} \\ Z U_{m}-U_{s}\end{array}\right)$.

Note that the blocking is now quite different from that used in Section 3. Here $U_{s}$ refers to slave interface variables, $U_{m}$ to master interface variables, $U_{i}$ to subregion interior variables, and $\Lambda$ to the Lagrange multipliers. The matrix $A_{i i}$ can be ordered by subregion and is block diagonal for such an ordering. Since several slave variables can be equated to a single master variable at cross points, the matrix $Z$ will not generally be an identity matrix; however, each row of $Z$ will be zero except for a single entry of one corresponding to a master variable.

We formally reorder (52) as

$\left(\begin{array}{cccc}A_{s s} & I & A_{s m} & A_{s i} \\ I & 0 & -Z & 0 \\ A_{m s} & -Z^{t} & A_{m m} & A_{m i} \\ A_{i s} & 0 & A_{i m} & A_{i i}\end{array}\right)\left(\begin{array}{c}\delta U_{s} \\ \Lambda \\ \delta U_{m} \\ \delta U_{i}\end{array}\right)=\left(\begin{array}{c}R_{s} \\ Z U_{m}-U_{s} \\ R_{m} \\ R_{i}\end{array}\right)$.

Block elimination of the slave variables and Lagrange multipliers leads to the reduced system

$$
\begin{gathered}
\left(\begin{array}{c}
A_{m m}+A_{m s} Z+Z^{t} A_{s m}+Z^{t} A_{s s} Z A_{m i}+Z^{t} A_{s i} \\
A_{i m}+A_{i s} Z
\end{array}\right)\left(\begin{array}{c}
\delta U_{m} \\
\delta U_{i}
\end{array}\right)= \\
\left(\begin{array}{c}
R_{m}+Z^{t} R_{s}-\left(A_{m s}+Z^{t} A_{s s}\right)\left(Z U_{m}-U_{s}\right) \\
R_{i}-A_{i s}\left(Z U_{m}-U_{s}\right)
\end{array}\right)
\end{gathered}
$$

The matrix appearing on the left-hand-side of (54) is the global stiffness matrix corresponding to the conforming finite element approximation. The term $R_{m}+Z^{t} R_{s}$ appearing on the right-hand-side corresponds to the usual residual for the conforming finite element approximation, and is independent of the choice of slave and master variables. However, the "jump" terms involving $Z U_{m}-U_{s}$ on the righthand-side of (54) do depend on the choice of master and slave variables.

We now consider the situation on a single processor, which we denote as processor $k, 1 \leq k \leq p$. We begin with a saddle point problem on subregion $k$ similar in structure to the global saddle point problem. This problem has the form

$$
\left(\begin{array}{cccc}
\bar{A}_{s s} & \bar{A}_{s m} & \bar{A}_{s i} & I \\
\bar{A}_{m s} & \bar{A}_{m m} & \bar{A}_{m i} & -\bar{Z}^{t} \\
\bar{A}_{i s} & \bar{A}_{i m} & \bar{A}_{i i} & 0 \\
I & -\bar{Z} & 0 & 0
\end{array}\right)\left(\begin{array}{c}
\delta \bar{U}_{s} \\
\delta \bar{U}_{m} \\
\delta \bar{U}_{i} \\
\Lambda
\end{array}\right)=\left(\begin{array}{c}
\bar{R}_{s} \\
\bar{R}_{m} \\
\bar{R}_{i} \\
\bar{Z} \bar{U}_{m}-\bar{U}_{s}
\end{array}\right) .
$$


Here the barred quantities refer to matrices and vectors for the local problem on subregion $k$. For example, the block diagonal matrix $\bar{A}_{i i}$ corresponds to the interior parts of the problem on processor $k$. The diagonal block $\bar{A}_{i i}$ arising from region $k$ is exactly the same as in the global saddle point problem (52). Since the remaining blocks correspond to coarse meshes, the overall order of $\bar{A}_{i i}$ is typically much smaller than $A_{i i}$. The residual $\bar{R}_{i}$ appearing on the right-handside of (55) has an interesting structure; for points lying in subregion $k$, it is the residual for the corresponding point in the global saddle point problem, and can be computed without communication on processor $k$. For points in the interior of $p-1$ coarse subregions, we set the residual to zero. If the local problems were all solved exactly, then the residual for the interior points would always be zero. In our case, we solve the local problems using the algebraic multilevel (multigraph) iterative method [12]. Thus, while the interior residuals will not be zero, we expect them to be much smaller than the residuals at the interface. By approximating interior residuals by zero in the coarse subregions, we avoid the need to communicate the interior residual values and to restrict them to the coarse mesh.

The interface equations are more interesting. An especially important point to emphasize here is that the designation of master and slave variables differs on each processor. The parts of the interface that involve subregion $k$ correspond exactly to the global saddle point problem; this is of course the most crucial point. The interface unknowns associated with subregion $k$ are all designated as the master unknowns for their mesh points, since they must be computed and updated as part of the solution process on processor $k$. The remaining interface points, lying on the interface of two or more subregions other than $k$ form a subset of the interface points of the global system. For these points we define the master and slave unknowns in an arbitrary fashion (in our code, we actually use an average).

The residuals $\bar{R}_{m}$ and $\bar{R}_{s}$ can be computed using the information contained in $R_{m}$ and $R_{s}$ in (52) under the assumption that residuals at interior points of the global fine mesh are all zero. (Note that calculating an entry of $\bar{R}_{m}$ or $\bar{R}_{S}$ at a coarse interface point involves expressing a coarse mesh residual as a linear combination of fine mesh residuals.) Also, the interface solution vectors $\bar{U}_{m}$ and $\bar{U}_{s}$ contain of subset of the values in $U_{m}$ and $U_{s}$ in (52). The parts of $R_{m}$ and $R_{s}$ corresponding to subregion $k$ are computed on processor $k$, and processor $k$ sends these residuals and the parts of $U_{m}$ and $U_{s}$ corresponding to subregion $k$ to all other processors. In turn, processor $k$ receives all other fine grid interface residuals and interface solution values from all other processors. This is accomplished in an all gather exchange in MPI. Following this exchange, each processor has all the values in $R_{s}, R_{m}, U_{s}$ and $U_{m}$, and from this information can extract the subset of information needed to form $\bar{R}_{s}, \bar{R}_{m}, \bar{U}_{s}$ and $\bar{U}_{m}$.
Block elimination of the slave variables and Lagrange multipliers in (55) leads to the reduced system

$$
\begin{gathered}
\left(\begin{array}{c}
\left.\bar{A}_{m m}+\bar{A}_{m s} \bar{Z}+\bar{Z}^{t} \bar{A}_{s m}+\bar{Z}^{t} \bar{A}_{s s} \bar{Z} \bar{A}_{m i}+\bar{Z}^{t} \bar{A}_{s i}\right)\left(\begin{array}{c}
\delta \bar{U}_{m} \\
\delta \bar{U}_{i}
\end{array}\right)= \\
\bar{A}_{i m}+\bar{A}_{i s} \bar{Z}
\end{array}\right) \\
\left(\begin{array}{c}
\bar{R}_{m}+\bar{Z}^{t} \bar{R}_{s}-\left(\bar{A}_{m s}+\bar{Z}^{t} \bar{A}_{s s}\right)\left(\bar{Z} \bar{U}_{m}-\bar{U}_{s}\right) \\
\bar{R}_{i}-\bar{A}_{i s}\left(\bar{Z}_{\bar{U}} \bar{U}_{m}-\bar{U}_{s}\right)
\end{array}\right)
\end{gathered}
$$

The system matrix appearing on the left hand side of (56) is the matrix used in the final adaptive refinement step on processor $k$, fine in subregion $k$ and coarse elsewhere, with possible modifications due to global fine mesh regularization. The right-hand-side can be computed once the exchange of interface data is complete. After the local system (56) is solved, the parts of $\delta \bar{U}_{m}$ and $\delta \bar{U}_{i}$ that correspond to subregion $k$ are extracted from the solution and used to update the global solution.

We note that the choice of master and slave unknowns for points on the coarse parts of the interface on processor $k$ is arbitrary. To resolve this ambiguity, in practice we take the master variable to be the average of all values that correspond to the interface point:

$U_{i_{m}} \equiv \frac{1}{\ell} \sum_{s=1}^{\ell} U_{i_{s}}$.

This is easy to do algorithmically, but awkward to describe in matrix notation. The effect is that the jump terms on the right-hand-side of (56) corresponding to coarse interface points are averaged over all choices of master variable. However, recall that for the interface points for subregion $k$, the master variable is always chosen to be the value from subregion $k$.

To summarize, a single domain decomposition/multigraph iteration on processor $k$ consists of:

1. locally compute $\bar{R}_{i}$ and parts of $R_{S}$ and $R_{m}$ associated with subregion $k$.

2. exchange boundary data, obtaining the complete fine mesh interface vectors $R_{m}, R_{s}, U_{m}$ and $U_{s}$.

3. locally compute the right-hand-side of (56) (using averages as described above).

4. locally solve (56) via the multigraph iteration.

5. update the fine grid solution for subregion $k$ using the appropriate parts of $\delta \bar{U}_{i}$ and $\delta \bar{U}_{m}$.

We close this section with some discussion of convergence criteria. This is a delicate issue, and there are several points to consider. First, in each DD iteration each processor (simultaneously) solves the largest linear system that is solved on that processor at any point during the calculation. Although these problems might be small in comparison with the size of the global system of linear equations, they still represent the most costly calculation in the entire adaptive procedure. Second, typically we have a very good initial guess given by (6). Third, the goal of the computation is to compute an approximate solution to the PDE, not an approximate solution to the linear system (of course the two are 
clearly related). Fourth, we expect to have very nonuniform adaptive meshes, and the norms used in the convergence criterion should take this into account.

We begin with a discussion of norms. Let $G_{i}$ denote the diagonal entry of the mass matrix corresponding to vertex $i$,

$G_{i}=\left\|\phi_{i}\right\|_{0, \Omega}^{2} \equiv \int_{\Omega} \phi_{i}^{2} d x$

where $\phi_{i}$ is the usual nodal basis function associated with vertex $i$ in the mesh. $G_{i}=\mathscr{O}\left(h_{i}^{2}\right)$, where $h_{i}$ is some measure of the size of elements sharing vertex $i$. Let $\mathscr{U}$ be a grid function; then

$\|\mathscr{U}\|_{G}^{2}=\sum_{i} \mathscr{U}_{i}^{2} G_{i}$

With this weighting, formally $\|\mathscr{U}\|_{G} \sim\left\|u_{h}\right\|_{0, \Omega}$, where $u_{h}$ is the finite element function corresponding to the grid function $\mathscr{U}$. Let $\mathscr{R}$ be a residual; then

$\|\mathscr{R}\|_{G^{-1}}^{2}=\sum_{i} \mathscr{R}_{i}^{2} G_{i}^{-1}$

With this weighting, intuitively $\|\mathscr{R}\|_{G^{-1}}$ looks like $\left\|e_{h}\right\|_{2, \Omega}$, where $e_{h}$ is the error in the finite element solution. This must only be formal since generally $e_{h} \notin \mathscr{H}^{2}(\Omega)$.

Norms are computed with respect to the global fine mesh; each processor computes its contribution to the global norm (the contribution from vertices in $\bar{\Omega}_{i}$ ) and then a communication step is necessary to form the global norm. The main convergence criterion is

$\frac{\left\|\boldsymbol{\delta} \mathscr{U}^{k}\right\|_{G}}{\left\|\mathscr{U}^{k}\right\|_{G}} \leq \max \left(\frac{\left\|\boldsymbol{\delta} \mathscr{U}^{0}\right\|_{G}}{\left\|\mathscr{U}^{0}\right\|_{G}}, \frac{\left\|\nabla e_{h}\right\|_{0, \Omega}}{\left\|\nabla u_{h}\right\|_{0, \Omega}}\right) \times 10^{-1}$.

Here $\mathscr{U}^{k}$ and $\delta \mathscr{U}^{k}$ are the global grid function and update, respectively, at iteration $k$, while $\left\|\nabla e_{h}\right\|_{0, \Omega}$ and $\left\|\nabla u_{h}\right\|_{0, \Omega}$ are the a posteriori error estimate and the initial solution (corresponding to grid function $\mathscr{U}^{0}$ ). In words, the iteration stops when the relative error in the solution is reduced by a factor of ten, or when the relative error in the solution of the linear system is a smaller by a factor of ten than the error in the PDE at the beginning of the iteration. The norm $\left\|\nabla e_{h}\right\|_{0, \Omega}$ appears instead of, e.g., $\left\|e_{h}\right\|_{0, \Omega}$ because it arises naturally in the context of a posteriori error estimation and it is the norm for which the strongest theoretical results are available. On the other hand, the use of different norms does introduce some inconsistency into (59). One could systematically replace $\|\cdot\|_{G}$ with $\|\nabla \cdot\|_{0, \Omega}$ at an increased computational cost in order to resolve the inconsistency should that prove necessary. It created no problems in the numerical experiments presented in this work. A secondary convergence criterion is

$\frac{\left\|\mathscr{R}^{k}\right\|_{G^{-1}}}{\left\|\mathscr{R}^{0}\right\|_{G^{-1}}} \leq 10^{-2}$

Typically, (59) is satisfied before (60).
Finally, on each processor the multigraph iterative method was used to solve local problems of the form (56). The convergence for the multigraph iteration was

$\frac{\left\|\overline{\mathscr{R}}^{j}\right\|_{\ell^{2}}}{\left\|\overline{\mathscr{R}}^{0}\right\|_{\ell^{2}}} \leq 10^{-4}$.

Here $\overline{\mathscr{R}}^{j}$ denotes the local residual at multigraph iteration $j$. The choice of $\|\cdot\|_{\ell^{2}}$ arose because the multigraph solver was part of a stand-alone package for solving linear systems [3] that was incorporated into PLTMG. As an algebraic multilevel method, it had no information about the linear system beyond the matrix and right hand side, and hence no basis to choose another norm. One could of course provide additional information and use another norm if necessary. The use of the more stringent tolerance $10^{-4}$ in (61) was to try to insure that the approximation of the interior residuals by zero at coarse grid points remained valid throughout the domain decomposition iteration.

\section{Numerical Results}

In this section, we present some numerical results. Our examples were run on a small LINUX-based Beowulf cluster, consisting of 20 dual 1800 Athlon-CPU nodes with 2GB of memory each, a dual Athlon file server, and a 100Mbit CISCO 2950G Ethernet switch. This cluster runs the NPACI ROCKS version of LINUX (based on RedHat 7.1), and employs MPICH1.2.2 as its MPI implementation. The computational kernels of PLTMG $[4,3]$ are written in FORTRAN; the g77 compiler (version 2.96) was used in these experiments, invoked using the script mpif77 and optimization flag - $O$.

In these experiments, we used PLTMG to solve the boundary value problem

$-\Delta u=1 \quad$ in $\Omega$,

$u=0 \quad$ on $\partial \Omega$,

where $\Omega$ is a domain shaped like Lake Superior.

In our first experiment, we computed an adaptive mesh with $N_{p}$ vertices on a single processor. This mesh was then broadcast to $p$ processors, where the variant strategy of combined coarsening and refinement was used to transfer approximately $N_{p} / 2$ vertices from outside $\Omega_{i}$ to inside $\Omega_{i}$. The global fine mesh was then made conforming as described in [8]. Note that the adaptive strategies implemented in PLTMG allow mesh moving and other modifications that yield meshes $\mathscr{T}_{i}$ that generally are not submeshes of the global conforming mesh $\mathscr{T}$. (Of course by definition they are identical on $\Omega_{i}$ and $\partial \Omega_{i}$.) However, PLTMG does insure that the partitions remain geometrically conforming, even in the coarse parts of the domain, and in particular, that the vertices on the interface system in each $\mathscr{T}_{i}$ are a subset of the vertices of interface system of the global mesh $\mathscr{T}$.

In this experiment, three values of $N_{p}(50 \mathrm{~K}, 75 \mathrm{~K}$, and $100 \mathrm{~K})$, and seven values of $p\left(2^{k}, 1 \leq k \leq 7\right)$ were used, 
yielding global fine meshes ranging in size form about $100 \mathrm{~K}$ to $6.2 \mathrm{M}$ unknowns. Because the meshes were adaptively created, in general they violated the quasi-uniformity assumption used in the theory. Also, the goal of relocating $N_{p} / 2$ vertices from outside $\Omega_{i}$ to inside $\Omega_{i}$ could not always be exactly satisfied for all processors, especially in the case $p=2$. Since our cluster had only 20 nodes, for larger values of $p$ we simulated the behavior of a larger cluster in the usual way, by allowing nodes to have multiple processes. The solution and the load balance for the case $N_{p}=100 K, p=32$ is shown in Figure 2.
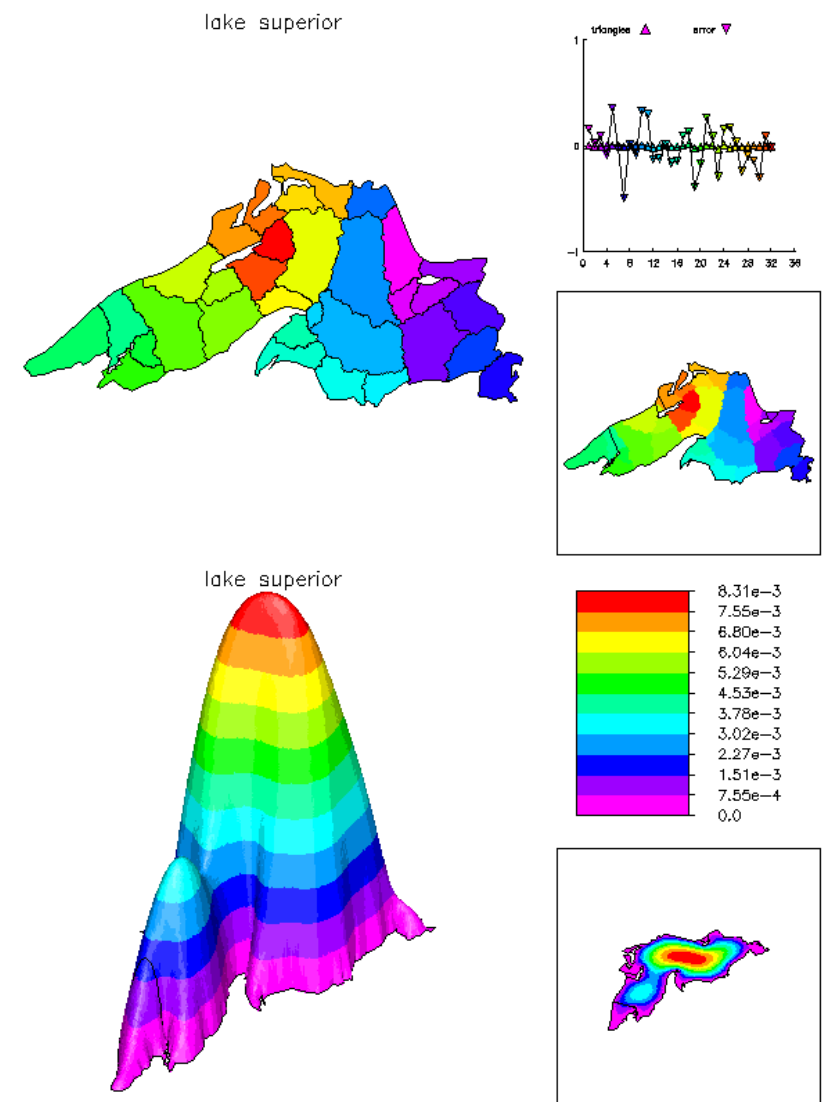

Fig. 2 The load balance (top) and solution (bottom) in the case $N_{p}=$ $100 K, p=32$.

Because our analysis suggests that the interface is especially important in our algorithm, in the process of regularizing the global fine mesh PLTMG also might make some additional refinement of coarse parts of the interface on each processor, simply to enhance the convergence rate of the following domain decomposition iteration. We define a graph $\mathscr{G}$ corresponding the the partition as follows. The nodes in the graph represent the subregions $\Omega_{i}$, and an edge $E_{i j}$ is present in the graph if and only if $\bar{\Omega}_{i} \cap \bar{\Omega}_{j} \neq \emptyset$. The distance $D_{i j}$ is defined as the number of edges in the shortest path connecting $\Omega_{i}$ to $\Omega_{j}$ in $\mathscr{G}$. In each coarse subregion $\Omega_{j}$, on the the interface $\Gamma \cap \partial \Omega_{j}$ we require the difference in refinement level between the local problem and the global problem to be bounded by $D_{i j}$. In words, the refinement on the parts of $\Gamma$ outside of $\bar{\Omega}_{i} \cap \Gamma$ is graded in proportion to the distance in the graph $\mathscr{G}$. This strategy concentrates some additional degrees of freedom on parts of $\Gamma$ where they are likely to have the greatest effect in terms of improving the rate of convergence. In this example, the amount of extra refinement for this strategy varied between none in the case $p=2$ to less than $10 \%$ for $p=128$. In Figure 3, we show the mesh density (local $h$ ) for the the case $N_{p}=100 k, p=32$ for the global mesh with $1.7 \mathrm{M}$ vertices. We also give an example of the local mesh for one processor. Here we see the mesh coincides with the global mesh on $\Omega_{i}$, and has larger elements elsewhere. We note that even with the requirement to maintain shape regularity, extra refinement along the interface is restricted to a very narrow band along the interface.

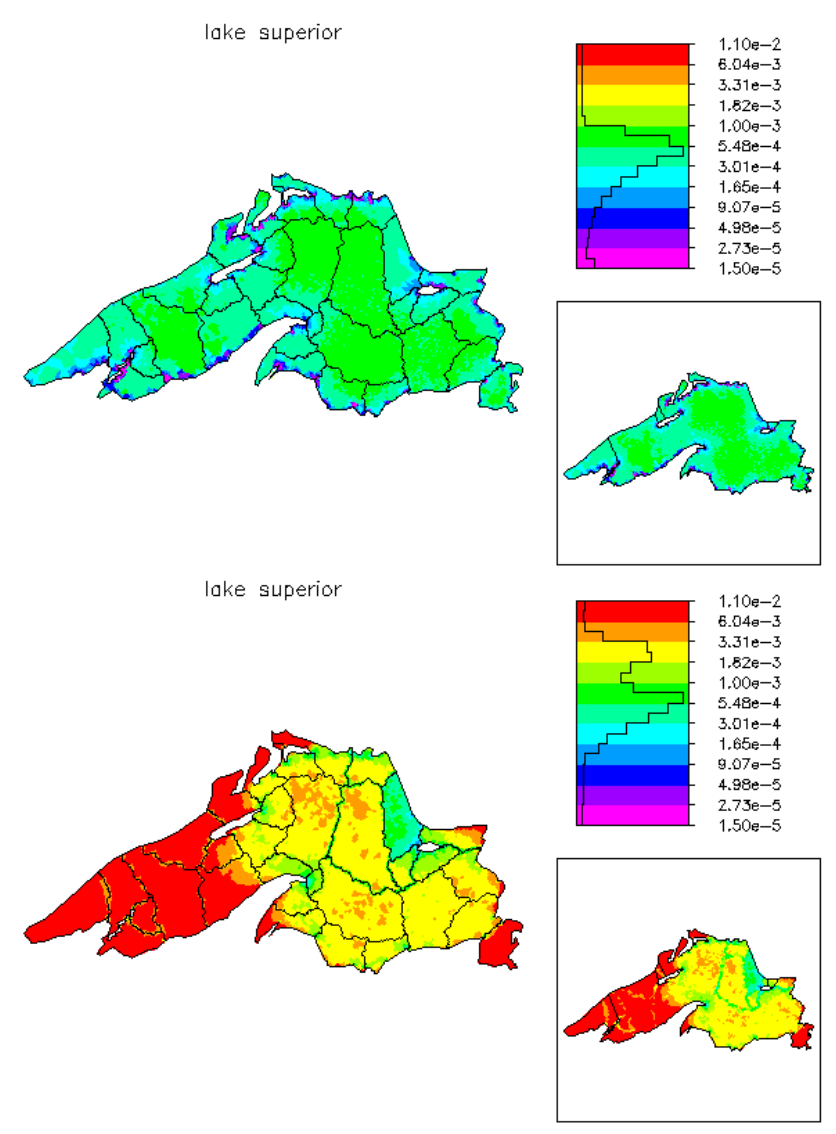

Fig. 3 The mesh density for the global mesh (top) and for one of the local meshes (bottom) in the case $N_{p}=100 K, p=32$.

In these experiments, we modified the convergence criterion described in Section 5 to

$\frac{\left\|\boldsymbol{\delta} \mathscr{U}^{k}\right\|_{G}}{\left\|\mathscr{U}^{k}\right\|_{G}} \leq \frac{\left\|\boldsymbol{\delta} \mathscr{U}^{0}\right\|_{G}}{\left\|\mathscr{U}^{0}\right\|_{G}} \times 10^{-4}$.

This is more stringent than necessary for purposes of computing an approximation to the solution of the partial differential equation, but it allows us to illustrate the behavior of 
the solver as an iterative method for solving linear systems of equations.

Table 1 summarizes this computation. The columns labeled $D D$ indicate the number of domain decomposition iterations required to satisfy the convergence criteria (62). For comparison, the number of iterations needed to satisfy the convergence criterion described in Section 5 is given in parentheses. From these results it is clear that the number of iterations is nearly constant over this range of $N$ and $p$, despite the fact that not all assumptions of the theory were satisfied. The size of the global mesh for the variant strategy can be estimated from the formula

$N \approx p \theta N_{p}+N_{p}$

for $\theta=1 / 2$. For $N_{p}=100 K, p=128,(63)$ predicts $N \approx$ 6300000 , where the observed $N=6263743$.

Table 1 Convergence Results for Variant Algorithm. Numbers of iterations needed to satisfy (62) are given in the column labeled DD. The numbers in parentheses are the number of iterations required to satisfy the convergence criterion described in Section 5.

\begin{tabular}{|r|rr|rr|rr|}
\hline & \multicolumn{2}{|c|}{$N_{p}=50 K$} & \multicolumn{2}{c|}{$N_{p}=75 K$} & \multicolumn{2}{c|}{$N_{p}=100 K$} \\
\hline$p$ & \multicolumn{1}{|c|}{$N$} & $D D$ & \multicolumn{1}{c|}{$N$} & \multicolumn{1}{c|}{$D D$} & \multicolumn{1}{c|}{$N$} & $D D$ \\
\hline 2 & 99107 & $10(3)$ & 148938 & $12(3)$ & 198861 & $7(3)$ \\
4 & 150108 & $7(3)$ & 225145 & $7(3)$ & 300166 & $6(2)$ \\
8 & 249613 & $7(3)$ & 374395 & $7(3)$ & 499269 & $6(2)$ \\
16 & 446250 & $7(3)$ & 670594 & $8(3)$ & 894626 & $8(3)$ \\
32 & 835266 & $7(3)$ & 1257664 & $7(3)$ & 1678726 & $7(3)$ \\
64 & 1599512 & $7(3)$ & 2413590 & $6(3)$ & 3226399 & $7(3)$ \\
128 & 3086008 & $6(3)$ & 4675761 & $7(3)$ & 6263743 & $7(3)$ \\
\hline
\end{tabular}

In our second experiment we solved the same problem using the original paradigm. On one processor, an adaptive mesh of size $N_{c}=10 \mathrm{~K}$ was created. This mesh was then partitioned into $p$ subregions, $p=2^{k}, 1 \leq k \leq 7$. This coarse mesh was broadcast to $p$ processors (simulated as needed) and each processor continued the adaptive process, creating a mesh of size $N_{p}$. We chose three values for $N_{p}, 50 \mathrm{~K}$, $75 \mathrm{~K}$, and $100 \mathrm{~K}$. This resulted in global meshes varying in size from approximately $90 \mathrm{~K}$ to $11 \mathrm{M}$ vertices. These global meshes were regularized, and a global DD solve was made as in the first experiment. As in the first experiment, the usual convergence criteria was replaced by (62) in order to illustrate the dependence of the convergence rate on $N$ and $p$. The results are summarized in Table 2 .

For the original paradigm the size of the global mesh is predicted by

$N \approx p N_{p}-(p-1) N_{c}$

Equation (64) predicts an upper bound, as it does not account for refinement outside of $\Omega_{i}$, needed to keep the mesh conforming and for other reasons. For example, for $N_{c}=10 \mathrm{~K}$, $N_{p}=100 \mathrm{~K}, p=128,(64)$ predicts $N \approx 11530000$ when actually $N=10921132$.
Table 2 Convergence Results for Original Algorithm. Numbers of iterations needed to satisfy (62) are given in the column labeled DD. The numbers in parentheses are the number of iterations required to satisfy the convergence criterion described in Section 5.

\begin{tabular}{|r|rc|rc|rc|}
\hline & \multicolumn{2}{|c|}{$N_{p}=50 K$} & \multicolumn{2}{|c|}{$N_{p}=75 K$} & \multicolumn{2}{|c|}{$N_{p}=100 K$} \\
\hline$p$ & \multicolumn{1}{|c|}{$N$} & $D D$ & \multicolumn{1}{|c|}{$N$} & $D D$ & \multicolumn{1}{|c|}{$D$} & $D D$ \\
\hline 2 & 89738 & $6(2)$ & 139599 & $6(2)$ & 189408 & $6(2)$ \\
4 & 168618 & $7(3)$ & 267831 & $7(3)$ & 367091 & $7(2)$ \\
8 & 324674 & $6(2)$ & 522879 & $7(3)$ & 721028 & $7(2)$ \\
16 & 630749 & $7(3)$ & 1026060 & $7(3)$ & 1421128 & $7(3)$ \\
32 & 1231557 & $8(3)$ & 2022028 & $7(3)$ & 2811006 & $7(3)$ \\
64 & 2397415 & $7(3)$ & 3975520 & $7(3)$ & 5551497 & $6(3)$ \\
128 & 4614399 & $8(3)$ & 7770798 & $7(3)$ & 10921132 & $8(3)$ \\
\hline
\end{tabular}

\section{References}

1. I. BABUŠKA AND J. M. MelenK, The partition of unity method, Internat. J. Numer. Methods Engrg., 40 (1997), pp. 727-758.

2. I. BABUŠKA AND V. NISTOR, Interior numerical approximation of boundary value problems with a distributional data, Numer. Methods Partial Differential Equations, 22 (2006), pp. 79-113.

3. R. E. BANK, Multigraph users' guide - version 1.0, tech. rep., Department of Mathematics, University of California at San Diego, 2001.

4. _ PLTMG: A software package for solving elliptic partial differential equations, users' guide 9.0, tech. rep., Department of Mathematics, University of California at San Diego, 2004.

5. - A domain decomposition solver for a parallel adaptive meshing paradigm, in Domain Decomposition Methods in Science and Engineering XVI, O. B. Widlund and D. E. Keyes, eds., vol. 55 of Lecture Notes in Computational Science and Engineering, Springer-Verlag, 2006, pp. 3-14.

6 . - Some variants of the Bank-Holst parallel adaptive meshing paradigm, Computing and Visualization in Science, 9 (2006), pp. 133-144.

7. R. E. BANK AND M. J. Holst, A new paradigm for parallel adaptive meshing algorithms, SIAM J. on Scientific Computing, 22 (2000), pp. 1411-1443.

8. A new paradigm for parallel adaptive meshing algorithms, SIAM Review, 45 (2003), pp. 292-323.

9. R. E. BANK AND P. K. JIMACK, A new parallel domain decomposition method for the adaptive finite element solution of elliptic partial differential equations, Concurrency and Computation: Practice and Experience, 13 (2001), pp. 327-350.

10. R. E. BANK, P. K. JiMACK, S. A. NADEEM, AND S. V. NePOMNYASCHIKH, A weakly overlapping domain decomposition preconditioner for the finite element solution of elliptic partial differential equations, SIAM J. on Scientific Computing, 23 (2002), pp. 1817-1841.

11. R. E. BANK AND S. LU, A domain decomposition solver for a parallel adaptive meshing paradigm, SIAM J. on Scientific Computing, 26 (2004), pp. 105-127 (electronic).

12. R. E. BANK AND R. K. SMITH, An algebraic multilevel multigraph algorithm, SIAM J. on Scientific Computing, 23 (2002), pp. 1572-1592.

13. F. BelgaCEM, The mortar finite element method with Lagrange multipliers, 1997. Preprint.

14. C. Bernardi, Y. MADAY, AND A. PATERA, A new nonconforming approach to domain decomposition: the mortar element method, in Nonlinear partial differential equations and their applications, H. Brezis and J. L. Lions, eds., Pitman Research Notes in Mathematics, New York, 1994, John Wiley and Sons, pp. 13-51.

15. D. BRAESS, Finite elements, Cambridge University Press, Cambridge, 2001 .

16. D. BRAESS, W. DAhMEN, AND C. Weiners, A multigrid algorithm for the mortar finite element method, SIAM J. on Numerical Analysis, 37 (1999), pp. 48-69. 
17. T. Chan And T. Matthew, Domain decomposition algorithms, in Acta Numerica, Cambridge University Press, 1994, pp. 61-143.

18. L. C. Evans, Partial differential equations, vol. 19 of Graduate Studies in Mathematics, American Mathematical Society, Providence, RI, 1998.

19. J. HUANG AND J. Zou, Construction of explicit extension operators on general finite element grids, Appl. Num. Math., 43 (2002), pp. 211-227.

20. J. HuAng AND J. Zou, A mortar element method for elliptic problems with discontinuous coefficients, IMA J. Numer. Anal., 22 (2002), pp. 549-576.

21. S. Lu, Parallel Adaptive Multigrid Algorithms, PhD thesis, Department of Mathematics, University of California at San Diego, 2004.

22. J. M. MELENK AND I. BABUŠKA, The partition of unity finite element method: basic theory and applications, Comput. Methods Appl. Mech. Engrg., 139 (1996), pp. 289-314.

23. W. MitChelL, The full domain partition approach to distributing adaptive grids, Applied Numerical Mathematics, 26 (1998), pp. 265-275.

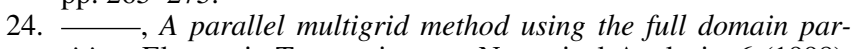
tition, Electronic Transactions on Numerical Analysis, 6 (1998), pp. 224-233.

25. W. F. MitCHELL, The full domain partition approach to parallel adaptive refinement, in Grid generation and adaptive algorithms (Minneapolis, MN, 1997), vol. 113 of IMA Vol. Math. Appl., New York, 1999, Springer, pp. 151-161.

26. J. A. NitsChe AND A. H. SChATZ, Interior estimates for RitzGalerkin methods, Math. Comp., 28 (1974), pp. 937-958.

27. B. SMith, P. BJORSTAD, AND W. GROPP, Domain Decomposition: Parallel Multilevel Methods for Elliptic Partial Differential Equations, Cambridge University Press, 1996.

28. G. Strang And G. J. FIX, An analysis of the finite element method, Prentice-Hall Inc., Englewood Cliffs, N. J., 1973.

29. A. Toselli AND O. Widlund, Domain decomposition methods-algorithms and theory, vol. 34 of Springer Series in Computational Mathematics, Springer-Verlag, Berlin, 2005.

30. L. B. WAHLBIN, Local behavior in finite element methods, in Handbook of numerical analysis, Vol. II, Handb. Numer. Anal., II, North-Holland, Amsterdam, 1991, pp. 353-522.

31. C. Wieners AND B. I. Wohlmuth, Duality estimates and multigrid analysis for saddle point problems arising from mortar discretizations, SIAM J. Sci. Comput., 24 (2003), pp. 2163-2184 (electronic).

32. B. I. Wohlmuth, A mortar finite element method using dual spaces for the Lagrange multiplier, SIAM J. Numer. Anal., 38 (2000), pp. 989-1012 (electronic).

33. $\mathrm{J} . \mathrm{XU}$, Iterative methods by space decomposition and subspace correction, SIAM Review, 34 (1992), pp. 581-613.

34. J. XU AND A. ZHOU, Local and parallel finite element algorithms based on two-grid discretizations, Math. Comp., 69 (2000), pp. 881-909. 